A. Kasue

Nagoya Math. J.

Vol. 114 (1989), 21-51

\title{
A CONVERGENCE THEOREM FOR RIEMANNIAN MANIFOLDS AND SOME APPLICATIONS
}

\author{
ATSUSHI KASUE
}

\section{§ 0. Introduction}

The purpose of the present paper is first to reformulate a Lipschitz convergence theorem for Riemannian manifolds originally introduced by Gromov [17] and secondly to give some applications of the theorem to a class of open Riemannian manifolds.

Let $\mathscr{M}(m, \Lambda, I, D)$ denote the class of compact $m$-dimensional Riemannian manifolds $M$ such that $\mid$ the sectional curvature $K_{M}$ of $M \mid \leq \Lambda^{2}$, the injectivity radius of $M \geq I>0$, and the diameter of $M \leq D$. The original Gromov's compactness theorem [17: Theorems 8.25 and 8.28] says that given a sequence $\left\{M_{n}\right\}$ in $\mathscr{M}(m, \Lambda, I, D)$, there exist a subsequence $\left\{M_{n^{\prime}}\right\}$ and a $C^{1,1}$ Riemannian manifold $M_{\infty}$ such that $\left\{M_{n^{\prime}}\right\}$ converges to $M_{\infty}$ in the Lipschitz distance (see [17] for the definitions of $C^{1,1}$ Riemannian manifolds and the convergence in the Lipschitz distance). Gromov gave an outline of an argument to justify this theorem. Later, Katsuda [27] worked out Gromov's proof in full detail. On the other hand, very recently, Peters [33] and Greene-Wu [16], independently, have improved the original version as above in a different manner. Both of them make use of harmonic coordinates and the Peter's argument in [32]. Since the Gromov's theorem appeared, some applications have been found by several authors (cf. e.g., [33], [28] and the literature).

In this paper, we shall also reformulate and prove the convergence theorem in our manner. Our proof is similar to the Peters' or GreeneWu's one, but more transparent than theirs. Our formulation of the convergence theorem is stated as follows:

Theorem A. Given a sequence $\left\{M_{n}\right\}_{n=1,2, \ldots}$ in $\mathscr{M}(m, \Lambda, I, D)$, there exist a subsequence $\left\{M_{n^{\prime}}\right\}$ of $\left\{M_{n}\right\}$, a smooth manifold $M_{\infty}$ and $C^{1, \beta}$ diffeomorphisms

Received August 24, 1987. 
$\Phi_{n^{\prime}}: M_{n^{\prime}} \rightarrow M_{\infty}(0<\beta<1)$ such that the pushforward $\Phi_{n^{\prime} * g_{n^{\prime}}}$ of the metric $g_{n^{\prime}}$ of $M_{n^{\prime}}$ converges to a metric $g_{\infty}$ of class $C^{1, \beta}$ in the $C^{1, \beta^{\prime}}$-topology $(0<$ $\left.\beta^{\prime}<\beta<1\right)$.

As is pointed out in [33], the regularity of the limit metric is optimal in terms of Hölder conditions.

The second half of this paper is devoted to give some applications of Theorem A (or its proof) to Riemannian manifolds of asymptotically nonnegative curvature. We call a complete connected, noncompact Riemannian manifold $M$ of asymptotically nonnegative curvature if the sectional curvature $K_{M}$ of $M$ satisfies

$$
K_{M} \geq-k \circ r_{o}
$$

where $r_{o}$ is the distance function to a fixed point $o$ of $M$ and $k(t)$ is a nonnegative, monotone nonincreasing function on $[0, \infty)$ such that the integral $\int^{\infty} t k(t) d t$ is finite. This class obviously contains the class of Riemannian open manifolds with nonnegative curvature everywhere. From the view point of geometry at infinity, it would be natural to study our class rather than the latter class. In [24], we have constructed a metric space $M(\infty)$ associated with a manifold $M$ of asymptotically nonnegative curvature. Let us here explain it briefly (see [24] for details). We call two rays $\sigma$ and $\gamma$ of $M$ equivalent if $\operatorname{dis}_{M}(\sigma(t), \gamma(t)) / t$ goes to zero as $t \rightarrow$ $\infty$. Define a distance $\delta_{\infty}$ on the equivalence classes by $\delta_{\infty}([\sigma],[\gamma]):=\lim _{t \rightarrow \infty}$ $d_{t}\left(\sigma \cap S_{t}, r \cap S_{t}\right) / t$ where $S_{t}$ denotes the metric sphere around a fixed point of radius $t$ and $d_{t}$ stands for the inner (or intrinsic) distance on $S_{t}$ induced from the distance $\operatorname{dis}_{M}($,$) on M$. Then we have a metric space $M(\infty)$ of the equivalence classes of rays with the distance $\delta_{\infty}$ which is independent of the choice of the fixed point (i.e., the center of $S_{t}$ ) and to which a family of scaled metric spheres $\left\{(1 / t) S_{t}\right\}$ converges with respect to the Hausdorff distance as $t$ goes to infinity. We note that the complement $M \backslash B_{t_{o}}$ of a metric ball $B_{t_{o}}$ with sufficiently large radius $t_{o}$ is homeomorphic to $S_{t_{o}} \times\left(t_{o}, \infty\right)$. Actually $M$ is isotopic to $B_{t_{o}}$ (for large $t_{o}$ ). For simplicity we call a connected component of $M \backslash B_{t}$ (for large $t$ ) an end of $M$ and denote it by $\mathscr{E}_{\alpha}(M)(\alpha=1, \cdots, \nu(M))$, where $\nu(M)$ is the number of the connected component of $M \backslash B_{t}$ or $S_{t}$ (for large $t$ ). We write $M_{\alpha}(\infty)$ for the connected component of $M(\infty)$ corresponding to $\mathscr{E}_{\alpha}(M)$, so that $\left\{(1 / t) S_{t} \cap \mathscr{E}_{\alpha}(M)\right\}$ converges to $M_{\alpha}(\infty)$ with respect to the 
Hausdorff distance as $t \rightarrow \infty$ and then $M_{\alpha}(\infty)$ turns out to be a compact inner metric space. Since $\operatorname{Vol}_{m-1}\left(S_{t} \cap \mathscr{E}_{\alpha}(M)\right) / t^{m-1}$ tends to a nonnegative constant as $t \rightarrow \infty$, let us write $\mathscr{V}_{o l_{m-1}}\left(M_{\alpha}(\infty)\right)$ for the limit. Although the metric spheres of $M$ around a fixed point are not smooth in general, we can approximate them by smooth hypersurfaces, e.g., the level hypersurfaces of the Riemannian convolution of the distance function to the fixed point. As is often the case, it is important to know the curvature of such smooth hypersurfaces. In Section 2, we shall prove the following

THEOREM B. Let $M$ be a manifold of asymptotically nonnegative curvature. Suppose that the sectional curvature $K_{M}$ of $M$ satisfies:

$$
\kappa_{M}:=\limsup _{t \rightarrow \infty} t^{2} K(t)<+\infty,
$$

where $K(t):=\sup \left\{\right.$ the sectional curvature of $M$ at points $x$ with $\operatorname{dis}_{M}(o, x)$ $\geq t\}$ and $o$ is a fixed point of $M$. Let $S_{t}$ be the metric sphere around $o$ of radius $t$. Then for large $t$, there exists a smooth hypersurface $S_{t}^{\prime}$ of $M$ which has the following properties:

(i ) $(1 / t) \max \left\{\max _{x \in S_{t}} \operatorname{dis}_{M}\left(x, S_{t}^{\prime}\right), \max _{y \in S_{t}^{\prime}} \operatorname{dis}_{M}\left(S_{t}, y\right)\right\} \rightarrow 0$ as $t \rightarrow \infty$.

(ii) There is a Lipschitz homeomorphism $\varphi_{t}: S_{t}^{\prime} \rightarrow S_{t}$ with

$$
e^{-\varepsilon(t)} \leq \frac{d_{t}\left(\varphi_{t}(x), \varphi_{t}(y)\right)}{d_{t}^{\prime}(x, y)} \leq e^{\varepsilon(t)}
$$

where $\varepsilon(t)$ goes to zero as $t \rightarrow \infty$ and $d_{t}$ (resp., $d_{t}^{\prime}$ ) denotes the inner distance on $S_{t}$ (resp. $\left.S_{t}^{\prime}\right)$.

(iii) The second fundamental form $\alpha_{t}^{\prime}$ of $S_{t}^{\prime}$ is estimated by

$$
\left\{-(1+a) \sqrt{\kappa_{M}} \tan a \sqrt{\kappa_{M}}-\varepsilon(t)\right\} g_{M} \leq t \alpha_{t}^{\prime} \leq\left\{1+\frac{1}{a}+\varepsilon(t)\right\} g_{M},
$$

where $a$ is a fixed constant with $0<a<\pi / 2 \sqrt{\kappa_{M}}$.

Moreover if $\mathscr{V}_{o l_{m-1}}\left(M_{\alpha}(\infty)\right)>0$, or equivalently $\lim _{t \rightarrow \infty} \operatorname{Vol}_{m}\left(B_{t} \cap\right.$ $\left.\mathscr{E}_{\alpha}(M)\right) / t^{m}>0$ for some end $\mathscr{E}_{\alpha}(M)$, then one has a smooth approximation $\hat{S}_{t}$ with (i) and (ii) as above, the second fundamental form $\hat{\alpha}_{t}$ of which enjoys the following property:

$$
(1-\varepsilon(t)) g_{M} \leq t \hat{\alpha}_{t} \leq(1+\varepsilon(t)) g_{M}
$$

on $\hat{S}_{t} \cap \mathscr{E}_{\alpha}(M)$.

Theorem B says in particular that under the additional condition 
(H.2) as above, $M(\infty)$ is the limit (with respect to the Hausdorff distance) of a family of compact $(m-1)$-dimensional Riemannian manifolds $\left\{M_{t}\right\}$ which are bounded uniformly in diameter and in curvature, and moreover when $\mathscr{V} l_{m-1}\left(M_{\alpha}(\infty)\right)>0$ for some $\alpha$, the volume of the connected component of $M_{t}$ converging to $M_{\alpha}(\infty)$ may be assumed to have a positive lower bound uniformly in $t$. Hence in this case (noncollapsing case), it turns out from Theorem A that $M_{\alpha}(\infty)$ is a smooth $(m-1)$-dimensional manifold with $C^{1, \beta}$-metric $(0<\beta<1)$. As for the other case (collapsing case), i.e., $\mathscr{V}_{o l_{m-1}}\left(M_{\alpha}(\infty)\right)=0$, we can apply the theory originated by Gromov and developed by Fukaya (cf. e.g., [12] and the literature) to our situation.

In the remaining sections, we shall show two applications of Theorem B. One of them, Theorem 3.2, is concerning the total curvature of a manifold of asymptotically nonnegative curvature and the other, Theorem 4.1, is on gap phenomena modeled after Euclidean space.

Theorem A is concerning a family of Riemannian manifolds in $\mathscr{M}(m, \Lambda, I, D)$. However a similar result is still valid for a family of Riemannian manifolds in $\mathscr{M}(m, \Lambda, I, \infty)$ (cf. $\S 1$ ). In this case, we should consider a pair $(M, p)$ of a Riemannian manifold $M$ and a point $p$ of $M$, and the topology of convergence in Theorem A should be appropriately modified, because $\mathscr{M}(m, \Lambda, I, \infty)$ may contain complete, noncompact Riemannian manifolds. This will be discussed elsewhere.

This paper is a revised version of a part of [23] which was completed while the author was a member of the Mathematical Sciences Research Institute at Berkeley. He greatly appreciates the institute for its hospitality.

\section{§1. Proof of Theorem A}

The purpose of this section is to prove Theorem A. Before going into the proof, we shall recall first some facts on harmonic coordinates. We refer the reader to e.g., Jost [21] and Greene-Wu [16] for details.

1.1. Let $M$ be a compact Riemannian manifold of dimension $m$ with Riemannian metric $g_{M}$. Given a point $o$ of $M$ and a unit tangent vector $u$ at $o$, we first define the almost linear function $\ell_{u}(x)$ associated with $u$ as follows: Let $r(x):=\operatorname{dis}_{M}(o, x), p(x):=\exp _{o} r(x) u, q(x):=\exp _{o}-r(x) u$ and then set $\ell_{u}(x):=\left\{\operatorname{dis}_{M}(x, q(x))^{2}-\operatorname{dis}_{M}(x, p(x))^{2}\right\} / 4 r(x)$. We assume 
from now that the sectional curvature $K_{M}$ of $M$ satisfies: $\left|K_{M}\right| \leq \Lambda^{2}$ for a constant $\Lambda$ and the injectivity radius of $M$ at $o \geq I$ for a constant $I>0$. Let $\delta$ be a number less that $\min \{I / 2, \pi / 4 \Lambda\}$ and let $\tilde{u}(x)$ be the vector at $x \in B_{\delta}(o)$ obtained by the parallel translation of $u$ from $o$ to $x$ along the radial geodesic. Then for each $x \in B_{\delta}(o)$, we have

(i ) $\left|\ell_{u}(x)\right| \leq r(x)$,

(ii) $\left|\operatorname{grad} \ell_{u}(x)-\tilde{u}(x)\right| \leq 2 \Lambda^{2}(\sinh 2 \Lambda r(x) / \sin 2 \Lambda r(x)) r^{2}(x)$,

(iii) $\left|\nabla^{2} b_{u}(x)\right| \leq\left\{9 \Lambda^{2}(\sinh 2 \Lambda r(x) / \sin 2 \Lambda r(x)) \Lambda r(x) \operatorname{coth} \Lambda r(x)\right\} r(x)$.

Let us take here an orthonormal basis $\left\{u_{1}, \cdots, u_{m}\right\}$ of $T_{o} M$ and set $\ell_{i}$ := $\ell_{u_{i}}(i=1, \cdots, m)$. Let $h_{i}: B_{\delta}(o) \rightarrow R(i=1, \cdots, m)$ be the solution of the Dirichlet problems: $\Delta h_{i}=0$ on $B_{\delta}(o)$ and $h_{i}=\ell_{i}$ on $\partial B_{\delta}(o)$, and define a harmonic map $\boldsymbol{H}: B_{\delta}(o) \rightarrow \boldsymbol{R}^{m}$ by $\boldsymbol{H}(x):=\left(h_{1}(x)-h_{1}(o), \cdots, h_{m}(x)-h_{m}(o)\right)$. Then we have the following

FACT 1.1. Let $m, \Lambda$ and $I$ be as before. Then there exists a positive constant $\delta(m, \Lambda, I)$ depending only on $m, \Lambda$, and $I$ that given any number $\delta \leq \delta(m, \Lambda, I)$, the above harmonic map $\boldsymbol{H}: B_{\delta}(o) \rightarrow \boldsymbol{R}^{m}$ defines a coordinate system around o which has the following properties:

(i ) $\left(1+\eta_{0}(m, \delta \Lambda)\right)^{-1} r(x) \leq|\boldsymbol{H}(x)| \leq\left(1+\eta_{0}(m, \delta \Lambda)\right) r(x)$

(ii) $\left(1+\eta_{0}(m, \delta \Lambda)\right)^{-1}|\xi|^{2} \leq \sum_{i, j=1}^{m} g_{i, j}(x) \xi^{i} \xi^{j} \leq\left(1+\eta_{0}(m, \delta \Lambda)\right)|\xi|^{2}$

(iii) $\left|g_{i, j}\right|_{C 1, \beta\left(B_{\delta}(o)\right)} \leq \eta_{1}(m, \Lambda, I, \beta)(0<\beta<1)$

where we set $g_{i, j}(x):=g_{M}\left(\nabla h_{i}, \nabla h_{j}\right)$. For any harmonic function $f$ on $B_{\delta}(o)$, one has

(iv) $|f|_{C^{2}, \beta\left(B_{\delta / 2}(o)\right)} \leq \eta_{2}(m, \Lambda, I, \beta) \sup _{B_{\delta}(o)}|f|$.

Moreover given an integer $k$ and a constant $\Lambda_{k}$, suppose that the norm of the $i$-th covariant derivatives $(0 \leq i \leq k)$ of the Ricci tensor of $M$ is bounded by $\Lambda_{k}$. Then one has

(v) $\left|g_{i, j}\right|_{\left.C^{1+k, \beta(B \delta}(o)\right)} \leq \eta_{3}\left(m, \Lambda, I, \beta, \Lambda_{k}\right)$

and for any harmonic function $f$ on $B_{\delta}(o)$,

(vi) $|f|_{C^{2+k, \beta\left(B_{\delta / 2}(o)\right)} \leq} \leq \eta_{4}\left(m, \Lambda, I, \beta, \Lambda_{k}\right) \sup _{B_{\delta}(o)}|f|$.

Here the constants $\eta_{i}(i=1, \cdots, 4)$ depend only on the given constants $m$, $\Lambda, I, \beta, k, \Lambda_{k}$ as indicated respectively.

1.2. Let us now prove Theorem $A$ in three steps.

Step 1. Let $M$ be a compact Riemannian manifold which belongs to $\mathscr{M}(m, \Lambda, I, D)$. Fix a positive constant $\delta$ such that $0<4 \delta<\delta(m, \Lambda, I)$, where $\delta(m, \Lambda, I)$ is as in Fact 1.1. Then we take a subset $\Gamma$ of finite points $p_{1}, \cdots, p_{\mu}$ of $M$ with $\operatorname{dis}_{M}\left(p_{i}, p_{j}\right)>2 \delta(i \neq j)$ and $\operatorname{dis}_{M}(\Gamma, p) \leq 4 \delta$ for any 
$p$ of $M$. By the Bishop's comparison theorem, we see that $\operatorname{Vol}_{m}\left(B_{\delta}\left(p_{i}\right)\right)$ $\geq c_{1}$ for some positive constant $c_{1}$ depending only on $m, \Lambda$, and $\delta$, and furthermore $\operatorname{Vol}_{m}(M) \leq c_{2}$ for some positive constant $c_{2}$ depending only on $m, \Lambda$ and $D$. Hence we have

$$
\mu c_{1} \leq \sum_{i=1}^{\mu} \operatorname{Vol}_{m}\left(B_{\delta}\left(p_{i}\right)\right) \leq \operatorname{Vol}_{m}(M) \leq c_{2} .
$$

This shows that the number $\mu$ as above is bounded by a constant $\mu_{\delta}$ depending only on $m, \Lambda, I, D$, and $\delta$. In what follows, for the sake of simplicity, we assume that the above $\mu$ is equal to $\mu_{\delta}$, by putting $p_{i}=p_{\mu}$ for $i: \mu \leq i \leq \mu_{\dot{\delta}}$.

Step 2. Set $\delta_{0}:=\delta(m, \Lambda, I), \eta_{0}:=\eta_{0}\left(m, \delta_{0} \Lambda\right), \delta_{1}:=10^{-1}\left(1+\eta_{0}\right)^{-8} \delta_{0}$, and $\delta_{k}:=\left(1+\eta_{0}\right)^{k} \delta_{1}(2 \leq k \leq 6)$, where $\delta(m, \Lambda, I)$ and $\eta_{0}\left(m, \delta_{0} \Lambda\right)$ are as in Fact 1.1. We take $\delta_{1} / 4$ as a fixed constant $\delta$ as in Step 1 and choose a subset $\Gamma$ of finite points $p_{1}, \cdots, p_{\mu}\left(\mu=\mu_{\delta_{1 / 4}}\right)$ of $M$ such that $\operatorname{dis}_{M}(\Gamma, p) \leq \delta_{1}$ for any $p \in M$. For each $i: 1 \leq i \leq \mu$, we have a harmonic coordinates $\boldsymbol{H}_{i}$ : $B_{\delta_{0}}\left(p_{i}\right) \rightarrow \boldsymbol{R}^{m}$ with the properties described in Fact 1.1. Observe first by Fact 1.1 that

$$
\boldsymbol{B}^{m}\left(\left(1+\eta_{0}\right)^{-1} \delta\right) \subset \boldsymbol{H}_{i}\left(B_{\delta}\left(p_{i}\right)\right) \subset \boldsymbol{B}^{m}\left(\left(1+\eta_{0}\right) \delta\right)
$$

for any $\delta: 0<\delta<\delta_{0}$, where $\boldsymbol{B}^{m}(t):=\left\{v \in \boldsymbol{R}^{m}:|v| \leq t\right\}$. Take a smooth function $\xi:[0, \infty) \rightarrow[0, \infty)$ such that $\xi(t) \equiv 1$ on $\left[0, \delta_{2}\right]$ and $\xi(t) \equiv 0$ on $\left[\delta_{3}, \infty\right)$. Set $\xi_{i}:=\xi\left(\left|\boldsymbol{H}_{i}\right|\right)(i=1, \cdots, \mu)$. Then each $\xi_{i}$ is a smooth function on $M$ such that the support of $\xi_{i}$ is contained in $B_{\delta_{6}}\left(p_{i}\right)$ and $\xi_{i} \equiv 1$ on $B_{\delta_{3}}\left(p_{i}\right)$. Let us now define a smooth map $\mathscr{H}_{M}: M \rightarrow R^{N}(N=(m+1) \mu)$ by $\mathscr{H}_{M}:=\left(\xi_{1} H_{1}, \cdots, \xi_{\mu} H_{\mu}, \xi_{1}, \cdots, \xi_{\mu}\right)$. Then $\mathscr{H}_{M}$ turns out to be a smooth embedding of $M$ into a ball $B^{N}\left(R_{0}\right)$ in $R^{N}$, where $R_{0}$ is a constant depending only on $m, \Lambda, I$, and $D$. We fix $i: 1 \leq i \leq \mu$, say $i=1$ for simplicity. Then $\mathscr{H}_{M}\left(B_{\delta_{3}}\left(p_{1}\right)\right)$ can be represented as a graph over $\boldsymbol{H}_{1}\left(B_{\delta_{3}}\left(p_{1}\right)\right)$, namely, $\mathscr{H}_{M}\left(B_{\delta_{3}}\left(p_{1}\right)\right)=\left\{\left(x, f_{2} \boldsymbol{F}_{2}, \cdots, f_{\mu} \boldsymbol{F}_{\mu}, f_{1}, \cdots, f_{\mu}\right): \boldsymbol{x}=\left(x_{1}, \cdots, x_{m}\right) \in \boldsymbol{H}_{1}\left(B_{\delta_{3}}\left(p_{1}\right)\right)\right\}$, where $\boldsymbol{F}_{j}=\boldsymbol{H}_{j} \circ \boldsymbol{H}_{1}^{-1}$ and $f_{j}=\xi\left(\left|\boldsymbol{F}_{j}\right|\right)$. Note here that $\boldsymbol{H}_{1}\left(B_{\delta_{1}}\left(p_{1}\right)\right) \subset \boldsymbol{B}^{m}\left(\delta_{2}\right) \subset$ $\boldsymbol{H}_{1}\left(B_{\hat{o}_{3}}\left(p_{1}\right)\right)$. Thus by Fact 1.1 , we see that for any $\beta: 0<\beta<1$, the $C^{2, \beta}$ norm of $\boldsymbol{F}_{j}$ and the $C^{2, \beta}$ norm of $\boldsymbol{F}_{j}$ and the $C^{2, \beta}$ norm of $f_{j}$ on $\boldsymbol{B}^{m}\left(\delta_{2}\right)$ are bounded from above by a constant $c_{3}$ depending only on $m, \Lambda, I$ and $\beta$ This implies in particular that the length of the second fundamental form $\alpha_{M}$ of the embedding $\mathscr{H}_{M}: M \rightarrow B^{N}\left(R_{0}\right)$ is bounded by a constant $c_{4}$ depending only on $m, \Lambda, I$ and $\beta$.

Step 3. Let $\left\{M_{n}\right\}$ be a sequence of Riemannian manifolds in 
$\mathscr{M}(m, \Lambda, I, D)$. We identity $M_{n}$ (resp., the metric $g_{n}$ on $M_{n}$ ) with the image

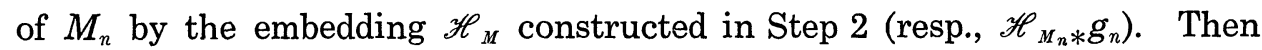
by the observations in Step 2, we have a subsequence $\left\{M_{n^{\prime}}\right\}$ of $\left\{M_{n}\right\}$ and a compact submanifold $M_{\infty}$ of class $C^{2, \beta}$ embedded in $B^{N}\left(R_{0}\right)$ such that $\left\{M_{n^{\prime}}\right\}$ converges to $M_{\infty}$ in the $C^{2, \beta^{\prime}}$ topology $\left(0<\beta^{\prime}<\beta<1\right)$ as $n^{\prime} \rightarrow \infty$. In what follows, we consider sufficiently large $n^{\prime}$ so that the projection $\Pi_{n^{\prime}}: M_{n^{\prime}} \rightarrow M_{\infty}$ along the normals to $M_{\infty}$ induces a $C^{2, \beta}$-diffeomorphism from $M_{n^{\prime}}$ onto $M_{\infty}$. Hence we have a sequence of $C^{1, \beta}$ metrics $\left\{\Pi_{n^{\prime} *} g_{n^{\prime}}\right\}$ on $M_{\infty}$. We claim here that, taking a subsequence $\{\hat{n}\}$ of $\left\{n^{\prime}\right\}$ if necessary,

(\#) $\left\{\Pi_{\hat{n} *} g_{\hat{n}}\right\}$ converges to a $C^{1, \beta}$ metric $g_{\infty}$ on $M_{\infty}$ in $C^{1, \beta^{\prime}}$ topology $\left(0<\beta^{\prime}<\beta<1\right)$ as $\hat{n} \rightarrow \infty$.

In fact, let $\left\{p_{n^{\prime}, i}\right\}_{i=1, \ldots, \mu}$ be as in Step 2 for $M_{n^{\prime}}$ and fix an index $i$, say $i=1$ for simplicity. Moreover let $\boldsymbol{P}$ (resp., $\boldsymbol{P}_{n^{\prime}}$ ) be the orthogonal projection from $\boldsymbol{R}^{N}$ onto $\boldsymbol{R}^{m}=\left\{\left(x_{1}, \cdots, x_{m}, 0, \cdots, 0\right) \in \boldsymbol{R}^{N}\right\}$ (resp., the restriction of $\boldsymbol{P}$ to the metric ball $B_{\delta_{3}}\left(p_{n^{\prime}, 1}\right)$ of $M_{n^{\prime}}$ around $p_{n^{\prime}, 1}$ with radius $\delta_{3}$ ). Define a $C^{2, \beta}$ diffeomorphism $\phi_{n^{\prime}}$ from $\boldsymbol{B}^{m}\left(\delta_{2}\right)$ into $\boldsymbol{R}^{m}$ by $\phi_{n^{\prime}}:=\boldsymbol{P} \circ \Pi_{n^{\prime}} \circ \boldsymbol{P}_{n^{\prime}}^{-1}$. Then the $C^{2, \beta}$-norm of $\phi_{n^{\prime}}$ is bounded uniformly in $n^{\prime}$, and hence $\phi_{n^{\prime}}$ converges to the identity map in $C^{2, \beta^{\prime}}$ topology $\left(0<\beta^{\prime}<\beta\right)$. We set $g_{n^{\prime} ; i, j}$ : = $\left(\boldsymbol{P}_{n^{\prime} *} g_{n^{\prime}}\right)\left(\partial / \partial x_{i}, \partial / \partial x_{j}\right)(i, j=1, \cdots, m)$. Then the $C^{1, \beta}$-norm of $g_{n^{\prime} ; i, j}$ on $\boldsymbol{B}^{m}\left(\delta_{2}\right)$ is bounded uniformly in $n^{\prime}$. Hence taking a subsequence $\left\{n^{\prime \prime}\right\}$ of $\left\{n^{\prime}\right\}$ if necessary, we see that as $n^{\prime \prime} \rightarrow \infty, \phi_{n^{\prime \prime} *} \boldsymbol{P}_{n^{\prime \prime} *} g_{n^{\prime \prime}}$ converges to a $C^{1, \beta}$ metric on $\boldsymbol{B}^{m}\left(\delta_{2}\right)$ in $C^{1, \beta^{\prime}}$ topology. Then it is easy to derive the above claim $(\sharp)$ from this observation. This completes the proof of Theorem A.

1.3. It would be of some use to restate Theorem A as follows:

TheOREm $\mathrm{A}^{\prime}$. Let $\left\{M_{n}\right\}$ be a sequence of Riemannian manifolds in $\mathscr{M}(m, \Lambda, I, D)$. Suppose that given a nonnegative integer $k$, the norm of the $i$-th covariant derivative $(0 \leq i \leq k)$ of the Ricci tensor on $M_{n}$ is bounded uniformly in $n$ by a constant $\Lambda_{k}$. Then there exist a subsequence $\left\{M_{n^{\prime}}\right\}$ of $\left\{M_{n}\right\}$, a smooth manifold $M_{\infty}$, and $C^{1+k, \beta}$-diffeomorphisms $\Phi_{n^{\prime}}: M_{n^{\prime}} \rightarrow M_{\infty}$ $(0<\beta<1)$ such that as $n^{\prime}$ goes to infinity, the pushforward of the metric of $M_{n^{\prime}}$ by $\Phi_{n^{\prime}}$ converges to a metric of class $C^{1+k, \beta}$ in $C^{1+k, \beta^{\prime}}$ topology $\left(0<\beta^{\prime}<\beta<1\right)$. Moreover, if in addition to the above assumptions, $\left\{M_{n}\right\}$ is a sequence of Kähler manifolds, i.e., $M_{n}=\left(M_{n}, g_{n}, J_{n}\right)$, then the pushforward of the almost complex structure $J_{n^{\prime}}$ of $M_{n^{\prime}}$ converges in $C^{1+k, \beta^{\prime}}$ topology to an integrable almost complex structure $J_{\infty}$ on $M_{\infty}$ which is 
parallel with respect to the limit metric on $M_{\infty}$.

Proof. The first part is a consequence of the argument of the proof for Theorem A and Fact 1.1(v). As for the second part, we observe that the $C^{1+k, \beta}$-norm of the almost complex structure $J_{n}$ on $M_{n}$ (with respect to the harmonic coordinates described in Fact 1.1) is bounded by a constant depending only on $m, \Lambda, I, D, \beta$ and $\Lambda_{k}$, since $J_{n}$ is parallel with respect to the metric of $M_{n}$. Therefore for a subsequence $\left\{n^{\prime}\right\}$ of $\{n\}$, $\left\{\Phi_{n^{\prime} *} J_{n^{\prime}}\right\}$ converges in $C^{1+k} \beta^{\prime}$ topology to an almost complex structure $J_{\infty}$ of class $C^{1+k, \beta}$ on $M_{\infty}$ as $n^{\prime} \rightarrow \infty$. Since $J_{n}$ is integrable, so is $J_{\infty}$ (cf. [30]) and moreover since $J_{n}$ is parallel with respect to the metric of $M_{n}$, so is $J_{\infty}$ with respect to the limit metric on $M_{\infty}$. This completes the proof of Theorem $\mathrm{A}^{\prime}$.

Let $M_{n^{\prime}}, g_{n^{\prime}}, M_{\infty}$ and $g_{\infty}$ be as in Theorem A. In the proof of Theorem A, we used the apriori estimates in Hölder spaces. If we apply the apriori estimates in Sobolev spaces, we see that the components of the limilt metric $g_{\infty}$, expressed in the harmonic coordinates discribed in Fact 1.1, are contained in the Sobolev spaces $W^{2, p}(p \geq 1)$. In particular, the curvature tensor $R_{\infty}$ of $g_{\infty}$ are almost everywhere defined (cf. [31] [33: Theorem 5.3]). Moreover the curvature tensor $R_{n^{\prime}}$ of $g_{n^{\prime}}$ converges weakly to $R_{\infty}$ as $n^{\prime} \rightarrow \infty$, namely, for any smooth covariant 4-tensor $T$, the inner product $\int_{M_{n^{\prime}}}\left\langle T, R_{n^{\prime}}\right\rangle=\int_{M_{n^{\prime}}} T^{i j k l} R_{n^{\prime} ; i j k l}$ converges to $\int_{M_{\infty}}\left\langle T, R_{\infty}\right\rangle$. This holds for the Ricci tensors or the scalar curvatures of $g_{n^{\prime}}$.

Let us now give an application of Theorem A. In order to state it, we need some notations. For a compact Riemannian manifold $M$ of dimension $m$ with metric $g_{M}$, we set

$$
\begin{aligned}
& \mathscr{R}(M):=\frac{1}{\operatorname{Vol}(M)^{(m-2) / m}} \int_{M}\left\|S_{M}-\frac{c(M)}{m} g_{M}\right\|, \\
& \mathscr{K}(M):=\frac{1}{\operatorname{Vol}(M)^{(m-2) / m}} \int_{M}\left\|R_{M}-\frac{c(M)}{m(m-1)} G_{M}\right\|, \\
& \mathscr{L}(M):=\frac{1}{\operatorname{Vol}(M)^{(m-3) / m}} \int_{M}\left\|\nabla R_{M}\right\|,
\end{aligned}
$$

where $S_{M}$ (resp. $R_{M}$ ) stands for the Ricci tensor of $M$ (resp. the curvature tensor of $M), G_{M}$ is a covariant 4-tensor defined by $G_{M}(W, Z, X, Y)=$ $g_{M}(W, X) g_{M}(Z, Y)-g_{M}(Z, X) g_{M}(Y, W)$, and $c(M)$ denotes the average of the scalar curvature $\rho_{M}$ of $M: c(M):=\int_{M} \rho_{M} / \operatorname{Vol}(M)$. 
THEOREm 1.1. Let $M$ be a Riemannian manifold in $\mathscr{M}(m, \Lambda, I, D)$ and $\delta$ a positive number. Then there exists a positive constant $\varepsilon(m, \Lambda, I, D ; \delta)$ depending only on $m, \Lambda, I, D$ and $\delta$, such that

(i) if $\mathscr{R}(M)<\varepsilon(m, \Lambda, I, D ; \delta)$, then $M$ is diffeomorphic to an Einstein manifold $M^{\prime}$ and the Lipschitz distance between $M$ and $M^{\prime}$ is less than $\delta$;

(ii) if $\mathscr{K}(M)<\varepsilon(m, \Lambda, I, D ; \delta)$, then $M$ is diffeomorphic to a space form $M^{\prime}$ of constant curvature and the Lipschitz distance between $M$ and $M^{\prime}$ is less than $\delta$;

(iii) if $\mathscr{L}(M)<\varepsilon(m, \Lambda, I, D ; \delta)$, then $M$ is diffeomorphic to a locally symmetric space $M^{\prime}$ and the Lipschitz distnce between $M$ and $M^{\prime}$ is less than $\delta$.

Theorem 1.1 is a consequence from the following

LEMmA 1.2. Let $M_{n^{\prime}}, g_{n^{\prime}}, M_{\infty}$ and $g_{\infty}$ be as in Theorem A.

(i) If $\mathscr{R}\left(M_{n^{\prime}}\right)$ goes to zero as $n^{\prime} \rightarrow \infty$, then $g_{\infty}$ is smooth and define an Einstein metric, i.e., the Ricci tensor $S_{\infty}$ of $g_{\infty}$ satisfies: $S_{\infty}=\left(c\left(M_{\infty}\right) / m\right), g_{\infty}$, where $c\left(M_{n^{\prime}}\right)$ converges to $c\left(M_{\infty}\right)$ as $n^{\prime} \rightarrow \infty$.

(ii) If $\mathscr{K}\left(M_{n^{\prime}}\right)$ goes to zero as $n^{\prime} \rightarrow \infty$, then $g_{\infty}$ is smooth and has constant curvature $c\left(M_{\infty}\right) / m(m-1)$.

(iii) If $\mathscr{L}\left(M_{n^{\prime}}\right)$ goes to zero as $n^{\prime} \rightarrow \infty$, then $g_{\infty}$ is smocth and $M_{\infty}$ is locally symmetric, i.e., the curvature tensor $R_{\infty}$ of $g_{\infty}$ is parallel.

Proof. Since $\left\{c\left(M_{n^{\prime}}\right)\right\}$ is a bounded sequence, we have a subsequence $\left\{c\left(M_{\hat{n}}\right)\right\}$ which converges to a constant $c$ as $\hat{n} \rightarrow \infty$. Observe first that the components $g_{n^{\prime}}^{i j}$ of the induced metric on the cotangent bundle $T^{*} M_{n^{\prime}}$, expressed in the harmonic coordinates described in Fact 1.1, satisfies

$$
\Delta_{n^{\prime}} g_{n^{\prime}}^{i j}=2 \sum_{k, \ell} g_{n^{\prime}}^{i k} g_{n^{\prime}}^{j \ell} S_{n^{\prime} ; k \ell}+2 \sum_{p, q, r, s} g_{n^{\prime}}^{p q} g_{n^{\prime}}^{r s} \Gamma_{n^{\prime} ; p r}^{i} \Gamma_{n^{\prime} ; q s}^{j}
$$

where $\Delta_{n^{\prime}}$ (resp. $\Gamma_{n^{\prime} ; p r}^{i}$ ) stands for the Laplacian of $g_{\infty}$ (resp. the Christoffel symbols of $\left.g_{\infty}\right)$. Suppose that $\mathscr{R}\left(M_{n^{\prime}}\right)$ goes to zero as $n^{\prime} \rightarrow \infty$. Then it follows that $g_{n^{\prime}}^{i j}$ satisfies weakly

$$
\Delta_{\infty} g_{\infty}^{i j}=\frac{2 c}{m} g_{\infty}^{i j}+2 \sum_{p, q, r, s} g_{\infty}^{p q} g_{\infty}^{r s} \Gamma_{\infty ; p r}^{i} \Gamma_{\infty ; q s}^{i} .
$$

Hence the standard regularity argument implies that $g_{\infty}^{i j}$ is smooth, and furthermore the Ricci tensor $S_{\infty}$ satisfies: $S_{\infty}=(c / m) g_{\infty}$. Moreover if $\mathscr{K}\left(M_{n^{\prime}}\right)$ goes to zero as $n^{\prime} \rightarrow \infty$, then $\mathscr{K}\left(M_{\infty}\right)=0$ and hence $M_{\infty}$ has constant curvature. Finally, let us assume that $\mathscr{L}\left(M_{n^{\prime}}\right)$ goes to zero as $n^{\prime}$. 
Since the covariant derivative $\nabla R_{n^{\prime}}$ of the curvature tensor $R_{n^{\prime}}$ of $M_{n^{\prime}}$ converges weakly to the covariant derivative $\nabla \cdot R_{\infty}$ (in the weak sense) of the curvature tensor $R_{\infty}$ of $M_{\infty}$ as $n^{\prime} \rightarrow \infty$, we see that $\nabla \cdot R_{\infty} \equiv 0$. Then it follows from the regularity argument that $g_{\infty}$ is smooth and $R_{\infty}$ is parallel. This completes the proof of Lemma 1.2.

\section{§2. Proof of Theorem $B$}

The purpose of this section is to prove Theorem B in Introduction. We shall carry out the proof, based on (the proof of) Theorem A and some facts given in [24]. Throughout this section, let us denote by $M$ a manifold of asymptotically nonnegative curvature. $r_{p}, B_{t}(p)$ and $S_{t}(p)$, respectively, stand for the distance to a point $p$ of $M$, the metric ball around $p$ of radius $t$ and the metric sphere around $p$ of radius $t$.

2.1. To begin with, we define a Lipschitz function $F_{p}: M \rightarrow R$ associated with a family of metric spheres $\left\{S_{t}(p)\right\}$ around a point $p$ of $M$ by $F_{p}(x):=\lim _{t \rightarrow \infty} t-\operatorname{dis}_{M}\left(x, S_{t}(p)\right)$. In order to prove Theorem B, we have to recall the following two facts:

FACT 2.1 ([24: Lemma 1.4]).

(i) For any fixed point $p$ of $M, F_{p}(x) / r_{p}(x)$ converges to 1 as $x$ goes to infinity. In particular, $F_{p}: M \rightarrow R$ is an exhaustion function on $M$, namely, $\left\{x \in M: F_{p}(x) \leq t\right\}$ is compact for any $t \in \boldsymbol{R}$.

(ii) As $x \in M$ goes to infinity,

$$
\begin{aligned}
& \max \left\{\Varangle(u, v): u, v \in \nabla \cdot r_{p}(x)\right\} \rightarrow 0, \\
& \max \left\{\Varangle(u, v): u \in \nabla \cdot r_{p}(x), v \in \nabla \cdot F_{p}(x)\right\} \rightarrow 0,
\end{aligned}
$$

where $\nabla \cdot r_{p}(x):=\left\{v \in T_{x} M:|v|=1, t+r_{p}\left(\exp _{x}-t v\right)=r_{p}(x)\left(0 \leq t \leq r_{p}(x)\right)\right\}$ and $\nabla \cdot F_{p}:=\left\{v \in T_{x} M:|v|=1, F_{p}\left(\exp _{x} t v\right)-t=F_{p}(x)(t \geq 0)\right\}$.

FACT 2.2 ([24: Lemma 1.5]). Let o be the base point in (H.1) and $J_{k}$ the solution of an equation: $J_{k}^{\prime \prime}+k J_{k}=0$, subject to the initial conditions: $J_{k}(0)=0$ and $J_{k}^{\prime}(0)=1$, where $k$ is as in (H.1). Then for any large $t \gg 0$ and small $\varepsilon>0$, there is a constant $\delta(t, \varepsilon)>0$ such that the Riemannian mollifier $r_{\delta}$ of $r\left(:=r_{o}\right)(0<\delta \leq \delta(t, \varepsilon))$ is well defined and smooth on $B_{t}(o)$, and it has the following properties:

(i ) $\left|r-r_{\delta}\right| \leq \varepsilon$,

(ii) $1-\varepsilon-\theta_{1}(r-\varepsilon) \leq\left|\nabla r_{\delta}\right| \leq 1+\varepsilon$,

(iii) $1-\varepsilon \leq\left|\nabla r_{j}\right|(x)$ if $\operatorname{dis}_{M}\left(x, \mathscr{E}_{0}\right) \geq \varepsilon$, 
(iv) $\nabla^{2} r_{\delta} \leq(1+\varepsilon)\left(\log J_{k}\right)^{\prime} \circ r_{\delta}$

where $\mathscr{E}_{0}$ stands for the cut locus of $M$ with respect to the base point o and $\theta_{1}(s):=\max \{\Varangle(u, v): u, v \in \nabla \cdot r(x), r(x) \geq s\}$. Moreover the Riemannian mollifier $F_{\delta}$ of $F\left(:=F_{0}\right)$ is also well defined and smooth on $B_{t}(o)$, and it satisfies:

(v) $\left|F-F_{o}\right| \leq \varepsilon$,

(vi) $\left|\nabla F_{\delta}-\nabla r_{\delta}\right| \leq \varepsilon+\theta_{2}(r-\varepsilon)$,

(vii) $\nabla^{2} F_{\delta} \geq-(1+\varepsilon) \int_{F}^{\infty} k(s) d s$ on $\left\{x \in B_{t}(o): F(x)>0\right\}$ if $k \neq 0$ near $+\infty$

$\nabla^{2} F_{\delta} \geq-\varepsilon-\int_{F}^{\infty} k(s) d s$ on $\left\{x \in B_{t}(o): F(x)>0\right\}$ if $k \equiv 0$ near $+\infty$, where $\theta_{2}(s):=\max \{\Varangle(u, v): u \in \nabla \cdot r(x), v \in \nabla \cdot F(x), r(x) \geq s\}$.

2.2. Let us now give the proof of Theorem $B$ which is devided into three steps. In what follows, we assume that $M$ satisfies (H.2) in Theorem B.

Step 1. Let $F\left(:=F_{0}\right)$ be the Lipschitz function associated with a family of the metric spheres $\left\{S_{t}(o)\right\}$ around a point, say the base point $o$ in (H.1). Let us fix sufficiently large numbers $T$ and $T^{\prime}$ with $T<T^{\prime}$ and sufficiently small numbers $\varepsilon, \delta(>0)$ with $\delta \leq \delta\left(T^{\prime}, \varepsilon\right)$ as in Fact 2.2. We consider the Riemannian mollifier $F_{\delta}$ of $F$ on $B_{T},(o)$, and set $\Omega_{\delta, T}:=$ $\left\{x \in M: F_{\delta}(x) \leq T\right\}$ and $\Sigma_{\delta, T}:=\partial \Omega_{\delta, T}$. For any $x \in \Sigma_{\delta, T}$, we denote by $\eta_{x}$ : $[0, \infty) \rightarrow M$ the geodesic which emanates from $x$ and which is tangent to the outer unit normal of $\Sigma_{\delta, T}$ at $x$. We define three numbers $\tau_{1}(x), \tau_{2}(x)$, and $\tau_{3}(x) \in(0, \infty]$ associated with $\eta_{x}$ as follows: $\tau_{1}(x):=\inf \{t \in(0, \infty)$ : $\left.\eta_{x}(t) \in \Omega_{\delta, T}\right\}, \tau_{2}(x):=\inf \left\{t \in(0, \infty): \Sigma_{\delta, T}\right.$ has no focal points along $\left.\eta_{x \mid[0, t]}\right\}$, and $\tau_{3}(x):=\sup \left\{t \in(0, \infty): \operatorname{dis}_{M}\left(\eta_{x}(s), \Sigma_{\delta, T}\right)=s\right.$ for $\left.s \in[0, t]\right\}$. Clearly, $\tau_{3}(x)$ $\leq \min \left\{\tau_{1}(x) / 2, \tau_{2}(x)\right\}$. For the sake of simplicity, we assume that $k$ is not constantly equal to zero. Then we have

$$
\tau_{3}(x) \geq \mu_{1}(T, \varepsilon):=\frac{T-\varepsilon}{\Lambda(T-\varepsilon)} \arctan \left[\frac{(1-\theta(T, \varepsilon)) \Lambda(T-\varepsilon)}{(1+\varepsilon) \lambda(T)}\right],
$$

where $\Lambda(t):=t \sqrt{K(t)}, K(t):=\sup$ the sectional curvature of $M$ at points $x$ with $r(x) \geq t\}, \lambda(t):=\int_{t}^{\infty} k(s) d s, \theta(t, \varepsilon):=2 \varepsilon+\theta_{1}(t-\varepsilon)+\theta_{2}(t-\varepsilon)$, and $\theta_{i}$ $(i=1,2)$ are as in Fact 2.2. Note that

$$
\frac{\mu_{1}(T, \varepsilon)}{T} \rightarrow \frac{\pi}{2 \sqrt{\kappa_{M}}}(\in(0, \infty])
$$


as $\varepsilon \rightarrow 0$ and $T \rightarrow \infty$. In order to prove (2.1), we first observe that the second fundamental form $\alpha_{\delta, T}$ of $\Sigma_{\delta, T}$ with respect to the outer unit normal satisfies

$$
\alpha_{\delta, T}=\frac{1}{\left|\nabla F_{\delta}\right|^{2}} \nabla^{2} F_{\delta} \geq-\frac{(1+\varepsilon)}{1-\theta(T, \varepsilon)} \int_{T}^{\infty} k(u) d u,
$$

because of Fact 2.2(vi), (vii). Let $J_{T, \varepsilon}$ be the solution of an equation: $J_{T, \varepsilon}^{\prime \prime}+(T-\varepsilon)^{-2} \Lambda^{2}(T-\varepsilon) J_{T, \varepsilon}=0$, subject to the initial conditions: $J_{T, \varepsilon}(0)$ $=1$ and $J_{T, \varepsilon}^{\prime}(0)=-(1+\varepsilon) \lambda(T) /\left(1-\theta_{3}(T, \varepsilon)\right)$, and set $\mu_{2}(T, \varepsilon):=\inf \{t>0$; $\left.J_{T, \varepsilon}(t)=0\right\}$. Then $\Sigma_{\delta, T}$ has no focal points along $\eta_{x \mid[0, t]}$ as long as $\eta_{x}([0, t])$ $\subset M \backslash \Omega_{\delta, T}$ and $t<\mu_{2}(T, \varepsilon)$, because we have (2.3) and the sectional curvature of $M$ is bounded from above by $(T-\varepsilon)^{-2} \Lambda^{2}(T-\varepsilon)$ on $M \backslash \Omega_{\delta, T}$. Since $\mu_{2}(T, \varepsilon) \geq \mu_{1}(T, \varepsilon)$, we have obtained

$$
\tau_{2}(x) \geq \mu_{1}(T, \varepsilon)
$$

if $\tau_{2}(x) \leq \tau_{1}(x)$. Suppose now that $\min \left\{\tau_{3}(x): x \in \Sigma_{\delta, T}\right\}<\mu_{1}(T, \varepsilon)$. Then it turns out from (2.4) that there exists a geodesic $\eta:[0,2 \ell] \rightarrow M$ such that $\ell=\min \left\{\tau_{3}(x): x \in \Sigma_{\delta, T}\right\}, \eta(t)=\eta_{x}(t)(t \in[0, \ell])$ for some $x \in \Sigma_{\delta, T}$, and $\eta(t)=$ $\eta_{y}(2 \ell-t)(t \in[\ell, 2 \ell])$ for some $y \in \Sigma_{\delta, T}$. Since we have by Fact 2.2 (vii): $d^{2} F_{\delta}(\eta(t)) / d t^{2} \geq-(1+\varepsilon) \int_{T}^{\infty} k(s) d s$ on $[0,2 \ell]$, it follows that $\left(F_{\delta} \circ \eta\right)^{\prime}(2 \ell)-$ $\left(F_{\delta} \circ \eta\right)^{\prime}(0) \geq-2 \ell(1+\varepsilon) \int_{T}^{\infty} k(s) d s$. This implies that

$$
\ell \geq\left[(1+\varepsilon) \int_{T}^{\infty} k(s) d s\right]^{-1} \geq \frac{T}{(1+\varepsilon) \lambda(T)} .
$$

On the other hand, $\ell$ is assumed to be less than $\mu_{1}(T, \varepsilon)$, and hence we have

$$
\frac{\Lambda(T-\varepsilon)}{(1+\varepsilon) \lambda(T)}<\arctan \frac{(1-\theta(T, \varepsilon)) \Lambda(T-\varepsilon)}{(1+\varepsilon) \lambda(T)}<\frac{(1-\theta(T, \varepsilon)) \Lambda(T-\varepsilon)}{(1+\varepsilon) \lambda(T)} .
$$

This is a contradiction. Thus we have shown (2.1).

Step 2. Fix positive constants $a, b$ with $a<b<\pi / 2 \sqrt{\kappa_{M}}(\leq+\infty)$. Then taking a sufficiently large number $T$ and a sufficiently small number $\varepsilon$, we may assume by (2.1) and (2.2) that $\min \left\{\tau_{3}(x): x \in \Sigma_{\delta, T}\right\}>b T$. Set $\rho_{\delta, T}$ $:=\operatorname{dis}_{M}\left(\Omega_{\delta, T}, *\right), \tilde{\Omega}_{\delta, T}:=\left\{x \in M: 0<\rho_{\delta, T}(x) \leq b T\right\}$ and $\tilde{\Sigma}_{\delta, T}:=\left\{x \in M: \rho_{\delta, T}(x)\right.$ $=a T\}$. Since $\rho_{\delta, T}$ is smooth on $\tilde{\Omega}_{\delta, T}, \tilde{\Sigma}_{\delta, T}$ is also smooth. Moreover it follows from (2.3) that 


$$
\nabla^{2} \rho_{\delta, T} \geq\left(\log J_{T, \varepsilon}\right)^{\prime} \circ \rho_{\delta, T}\left(g_{M}-d \rho_{\delta, T}^{2}\right)
$$

on $\tilde{\Omega}_{\delta, T}$, where $J_{T, \varepsilon}$ is as in Step 1. In particular, the second fundamental form $\tilde{\alpha}_{\delta, T}$ of $\tilde{\Sigma}_{\delta, T}$ satisfies

$$
\tilde{\alpha}_{\tilde{\partial}, T} \geq \mu_{3}(T, \varepsilon, a) g_{M}\left(\mu_{3}(T, \varepsilon, a):=\left(\log J_{T, \varepsilon}\right)^{\prime}(a T)\right) .
$$

Note that

$$
(1+a) T_{\mu_{3}}(T, \varepsilon, a) \rightarrow-(1+a) \sqrt{\kappa_{\mu}} \tan a \sqrt{\kappa_{\mu}}
$$

as $\varepsilon \rightarrow 0$ and $T \rightarrow \infty$. In order to get an upper bound of $\tilde{\alpha}_{\tilde{s}, T}$, we compare $\rho_{\delta, T}$ with $r\left(=r_{o}\right)$. Since

$$
\begin{aligned}
\frac{d}{d t} F_{\delta}\left(\eta_{x}(t)\right) & =1+\int_{0}^{t} \frac{d^{2}}{d s^{2}} F_{\delta}\left(\eta_{x}(s)\right) d s \\
& \left.\geq 1-(1+\varepsilon) \int_{T}^{\infty} k(s) d s \cdot t \quad \text { (by Fact } 2.2(\text { vii })\right) \\
& \geq 1-(1+\varepsilon) \int_{T}^{\infty} s k(s) d s \cdot \frac{t}{T} \\
& \geq 1-(1+\varepsilon) v \lambda(T)
\end{aligned}
$$

for any $x \in \Sigma_{\delta, T}$ and $t \in[0, b T]$, we see that

$$
\left\langle\nabla F_{\delta}, \nabla \rho_{\delta, T}\right\rangle \geq 1-(1+\varepsilon) b \lambda(T)
$$

on $\tilde{\Omega}_{\delta, T}$. In particular, we have by Facts 2.1 and 2.2

(2.8) $\min \left\{\left\langle\nabla \rho_{\delta, T}, v\right\rangle: v \in \nabla \cdot r(x), x \in \Omega_{\delta, T}\right\} \geq 1-(1+\varepsilon) b \lambda(T)-\varepsilon_{1}(T)$

where $\varepsilon_{1}(T)$ goes to zero as $T \rightarrow \infty$. Moreover it follows from (2.7) that

$$
F_{\delta} \geq T+\{1-(1+\varepsilon) s \lambda(T)\} \rho_{\delta, T}
$$

and hence

$$
r \geq T-\varepsilon+\{1-(1+\varepsilon) b \lambda(T)\} \rho_{\delta, T}
$$

on $\tilde{\Omega}_{\delta, T}$, since $F_{\delta} \leq F+\varepsilon \leq r+\varepsilon$. On the other hand, by Fact (2.1(i)), we have

$$
r \leq \rho_{\tilde{\delta}, T}+\left(1+\varepsilon_{2}(T)\right)(T+\varepsilon)
$$

where $\varepsilon_{2}(T)$ goes to zero as $T \rightarrow \infty$. It turns out from (2.9) that

$$
\nabla^{2} \rho_{\delta, T}<\left[\frac{1}{\rho_{\delta, T}}+\frac{\lambda(T-\varepsilon)}{(T-\varepsilon)\{1-(1+\varepsilon) b \lambda(T)\}}\right]\left(g_{\mu}-d \rho_{\delta, T}^{2}\right)
$$


on $\tilde{\Omega}_{\delta, T}$. In fact, by (2.9), we see that the sectional curvature at $\eta_{x}(t) \geq$ $-k\left(r\left(\eta_{x}(t)\right)\right) \geq-k(T-\varepsilon+\{1-(1+\varepsilon) b \lambda(T)\} t)$. Let $\hat{J}_{T, \varepsilon}$ be the solution of an equation: $\hat{J}_{T, \varepsilon}^{\prime \prime}-k(T-\varepsilon+\{1-(1+\varepsilon) b \lambda(T)\} t) \hat{J}_{T, \varepsilon}=0$, with $\hat{J}_{T, \varepsilon}(0)$ $=0$ and $\hat{J}_{T, \varepsilon}^{\prime}(0)=1$. Then we have

$$
\nabla^{2} \rho_{\delta, T}<\left(\log \hat{J}_{T, \varepsilon}\right)^{\prime} \circ \rho_{\delta, T}\left(g_{M}-d \rho_{\delta, T}^{2}\right)
$$

and

$$
\begin{aligned}
\left(\log \hat{J}_{T, \varepsilon}\right)^{\prime}(t) & =\frac{1}{\hat{J}_{T, \varepsilon}(t)}\left[1+\int_{0}^{t} k(T-\varepsilon+\{1-(1+\varepsilon) b \lambda(T)\} s) \hat{J}_{T, \varepsilon}(s) d s\right] \\
& \leq \frac{1}{\hat{J}_{T, \varepsilon}(t)}\left[1+\hat{J}_{T, \varepsilon}(t) \int_{0}^{t} k(T-\varepsilon+\{1-(1+\varepsilon) b \lambda(T)\} s) d s\right] \\
& \leq \frac{1}{t}+\frac{\lambda(T-\varepsilon)}{(T-\varepsilon)\{1-(1+\varepsilon) b \lambda(T)\}} .
\end{aligned}
$$

In particular, (2.11) implies that

$$
\hat{\alpha}_{\delta, T}<\mu_{4}(T, \varepsilon, a) g_{M}\left(\mu_{4}(T, \varepsilon, a):=\frac{1}{a T}+\frac{\lambda(T-\varepsilon)}{(T-\varepsilon)\{1-(1+\varepsilon) b \lambda(T)\}}\right) .
$$

Note that

$$
(1+a) T \mu_{4}(T, \varepsilon, a) \rightarrow 1+\frac{1}{a}
$$

as $\varepsilon \rightarrow 0$ and $T \rightarrow \infty$. Finally, let us summarize the results obtained above. For any large number $T$, we choose sufficiently small $\delta_{T}>0$ with $\lim _{T \rightarrow \infty} \delta_{T}=0$ and set $\tilde{S}_{T}:=\tilde{\Sigma}_{\delta_{\hat{T}}, \hat{T}}(\hat{T}:=T /(1+a))$. Then we have

(i) $\max \left\{\max _{x \in S_{T}(o)} \operatorname{dis}_{M}\left(x, \tilde{S}_{T}\right), \max _{y \in \tilde{S}_{T}} \operatorname{dis}_{M}\left(S_{T}(o), y\right)\right\} / T \rightarrow 0$ as $T$ goes to infinity (cf. (2.9) and (2.10));

(ii) the integral curve of $r_{\delta_{T}} /\left|\nabla r_{\delta_{T}}\right|^{2}$ defines a Lispchitz homeomorphism $\varphi_{T}$ from $\tilde{S}_{T}$ onto $S_{T}(o)$ such that

$$
e^{-\varepsilon(T)} \leq \frac{d_{T}\left(\varphi_{T}(x), \varphi_{T}(y)\right)}{\tilde{d}_{T}(x, y)} \leq e^{\varepsilon(T)}
$$

where $\varepsilon(T)$ goes to zero as $T \rightarrow \infty$ and $\tilde{d}_{T}$ (resp., $d_{T}$ ) denotes the inner distance on $\tilde{S}_{T}$ (resp., $S_{T}(o)$ ) (cf. (2.8) and Fact 2.1);

(iii) the second fundamental form $\tilde{\alpha}_{T}$ of $\tilde{S}_{T}$ in $M$ satisfies

$$
(1+a) \sqrt{\kappa_{M}} \tan a \sqrt{\kappa_{M}}-\varepsilon(T) \leq T \tilde{\alpha}_{T} \leq 1+\frac{1}{a}+\varepsilon(T),
$$


where $a$ is a constant with $0<a<\pi / 2 \sqrt{\kappa_{M}}$ (cf. (2.5) and (2.12)). Thus we have proved the first assertion of Theorem B.

Step 3. We are now in the position to prove the second assertion of the theorem, by applying Theorem A to our situation. Suppose that $\operatorname{Vol}_{m}\left(B_{t}(o) \cap \mathscr{E}_{\alpha}(M)\right) / t^{m}$ is bounded away from zero as $t \rightarrow \infty$, for some end $\mathscr{E}_{\alpha}(M)$ of $M$. This is equivalent to saying that

$$
\begin{aligned}
& \mathscr{V} o l_{m-1}\left(M_{\alpha}(\infty)\right): \stackrel{\text { (def.) }}{=} \lim _{t \rightarrow \infty} \operatorname{Vol}_{m-1}\left(S_{t}(o) \cap \mathscr{E}_{\alpha}(M)\right) / t^{m-1} \\
&=\lim _{t \rightarrow \infty} \operatorname{Vol}_{m-1}\left(\tilde{S}_{t} \cap \mathscr{E}_{\alpha}(M)\right) / t^{m-1} \\
&>0
\end{aligned}
$$

where $\left\{\tilde{S}_{t}\right\}$ is as in Step 2. For the sake of simplicity, we assume that $M$ has one end, so that $\mathscr{V} o l_{m-1}(M(\infty))$ is positive. Then if the dimension $m$ of $M$ is greater than or equal to 3 , the family $\left\{(1 / t) \tilde{S}_{t}\right\}$ of compact Riemannian manifolds is bounded uniformly in curvature, diameter and volume, and hence it turns out from Theorem $\mathrm{A}$ that when $m \geq 3, M(\infty)$ is a smooth manifold with a Riemannian metric of class $C^{1, \beta}(0<\beta<1)$, since $M(\infty)$ is the limit of $\left\{(1 / t) \tilde{S}_{t}\right\}$ with respect to the Hausdorff distance. In order to construct smooth approximations $\left\{\tilde{S}_{t}\right\}$ stated in the second assertion of Theorem $\mathrm{B}$, we first observe that the injectivity radius of $M$ at a point $x$ is bounded from below by $\operatorname{cr}(x)$ for some positive constant $c$. Actually, this follows from the argument in the proof of the first assertion of the theorem. Secondly, let us consider a family of Riemannian manifolds $\{(1 / t) M\}$ and set $A_{t}(a, b):==\left\{x \in(1 / t) M: b \leq \operatorname{dis}_{t}(o, x) \leq a\right\}$, where $\operatorname{dis}_{t}(o, x):=(1 / t) \operatorname{dis}_{M}(o, x)$. Then we have the following

Lemma 2.3. Fix two positive numbers $a, b$ with $a>b$. Then for large $t$, there exists a $C^{2, \beta}$-diffeomorphism $\Pi_{t}(0<\beta<1)$ from $A_{t}(a, b)$ into the cone $\mathscr{C}(M(\infty))$ over $M(\infty)$, i.e., $\mathscr{C}(M(\infty)):=[0, \infty) \times{ }_{t_{2}} M(\infty)$ which has the following properties: as $t$ goes to infinity, $\Pi_{t}\left(A_{t}(a, b)\right)$ converges to $[a, b]$ $\times_{t_{2}} M(\infty)$ and $\Pi_{t *} G_{t}$ converges to the metric $d t^{2}+t^{2} g_{\infty}$ in $C^{1, \beta}$ topology $\left(0<\beta^{\prime}<\beta<1\right)$, where $G_{t}$ (resp., $g_{\infty}$ ) is the Riemannian metric of $(1 / t) M$ (resp., $M(\infty))$.

The second assertion of Theorem $B$ is an immediate consequence of Lemma 2.3. Actually, (after fixing constants $a, b$ as above with $b<1<$ $a$ )) we can take the hypersurfaces $\hat{S}_{t}:=\Pi_{t}^{-1}(\{1\} \times M(\infty))$ as required approximations for the metric spheres $S_{t}(o)$. 
Proof of Lemma 2.3. We first observe that the injectivity radius of $(1 / t) M$ on $A_{t}(10 a, b / 10)$ is bounded from below by a positive constant $c$ (independent of $t$ ) and the sectional curvature $K_{t}$ of $(1 / t) M$ (for large $t$ ) is pinched as follows: $-\varepsilon(t) \leq K_{t} \leq 10^{4} b^{-2} \kappa_{M}$, where $\varepsilon(t)$ goes to zero as $t \rightarrow \infty$. Given a small positive constant $\delta<10^{-2} b$, we take a finite number of points $x_{t, 1}, \cdots, x_{t, \mu}$ of $A_{t}(4 a, b / 4)$ (for large $t$ ) such that $\operatorname{dis}_{t}\left(x_{t, i}, x_{t, j}\right)$ $\geq 2 \delta(i \neq j), \operatorname{dis}_{t}\left(x_{t, i}, o\right)>b / 10+2 \delta$, and the union of the metric balls $B_{t, 2 \delta}\left(x_{t, i}\right)$ of $(1 / t) M$ around $x_{t, i}$ of radius $2 \delta$ covers $A_{t}(2 a, b / 2)$. Then the number $\mu$ is bounded from above by a positive constant $\mu_{0}$ which is independent of $t$. In fact, by setting $A:=10 b^{-1} \sqrt{\kappa_{M}}$, we have

$$
\begin{aligned}
\mu \omega_{m-1} \int_{0}^{o}\left[\frac{\sin \Lambda u}{\Lambda}\right]^{m-1} d u & \leq \operatorname{Vol}_{m}\left(\bigcup_{1 \leq i \leq \mu} B_{t, \delta}\left(x_{t, i}\right)\right) \\
& \left.\leq \frac{1}{t^{m}} \operatorname{Vol}_{m}\left(B_{6 a t} / o\right)\right) \\
& \leq \frac{\omega_{m-1}}{t^{m}} \int_{0}^{6 a t} J_{k}^{m-1}(u) d u \quad \text { (cf. [24: Lemma 1.2]) } \\
& \leq \frac{\omega_{m-1}}{t^{m}} \int_{0}^{6 a t}\left(J_{k}^{\prime}(\infty) u\right)^{m-1} d u \\
& \leq \frac{1}{m} \omega_{m-1}\left(J_{k}^{\prime}(\infty)\right)^{m-1}(6 a)^{m}
\end{aligned}
$$

where $J_{k}$ is as in Fact 2.2 and $J_{k}^{\prime}(\infty):=\lim _{t \rightarrow \infty} J_{k}^{\prime}(t)\left(<\exp \int_{0}^{\infty} t k(t) d t\right)$ (cf. [14: Theorem C])). Thus we can apply the argument of the proof of Theorem A to our situation and obtain the maps $\Pi_{t}: A_{t}(a, b) \rightarrow \mathscr{C}(M(\infty)$ ) (for large $t$ ) mentioned in Lemma 2.3, since $A_{t}(a, b)$ converges to $[a, b] \times{ }_{t 2} M(\infty)$ with respect to the Hausdorff distance as $t$ goes to infinity. This completes the proof of Lemma 2.3.

2.3. Let us now give two corollaries to Theorem B.

Corllary 2.4. Let $M$ be as in Theorem B. Suppose that the dimension $m$ of $M$ is greater than or equal to 3 and, for some end $\mathscr{E}_{a}(M)$ of $M$, $\mathscr{V}_{o l-1}\left(M_{\alpha}(\infty)\right)$ is positive. Then:

(i) $M_{\alpha}(\infty)$ is a compact smooth manifold of dimension $m-1$ with $C^{1, \beta}$ metric $(0<\beta<1)$ such that the diameter diam $\left(M_{\alpha}(\infty)\right)$ is not greater than $\pi$ and the volume $\operatorname{Vol}_{m-1}\left(M_{\alpha}(\infty)\right)$ is equal to $\mathscr{V}_{o l-1}\left(M_{a}(\infty)\right)$ and not greater than the volume of a metric ball with radius $\operatorname{diam}\left(M_{\alpha}(\infty)\right)$ in the 
sphere of constant curvature 1.

(ii) If $\kappa_{M}<3$ and $M_{a}(\infty)$ is simply connected, or if $m$ is odd and $M_{a}(\infty)$ is orientable, then the injectivity radius of $M_{\alpha}(\infty)$ is not less than $\pi / \sqrt{1+\kappa_{M}}$ and $\operatorname{Vol}_{m-1}\left(M_{\alpha}(\infty)\right)$ is nct less than the volume of a metric ball with radius $\pi / \sqrt{1+\kappa_{M}}$ in the sphere of constant curvature $1+\kappa_{M}$.

(iii) If $\operatorname{diam}\left(M_{\alpha}(\infty)\right) \geq \pi / 2, \operatorname{Vol}_{m-1}\left(M_{\alpha}(\infty)\right)$ is not less than the volume of a metric ball with radius $\pi / 2 \sqrt{1+\kappa_{M}}$ in the sphere of constant curvature $1+\kappa_{M}$.

Proof. Since $M_{\alpha}(\infty)$ is the limit (in $C^{1, \beta^{\prime}}$ topology) of a family of compact Riemannian manifolds $\left\{\hat{S}_{t}\right\}$ such that their diameters are uniformly bounded from above, their volumes are uniformly bounded from below by a positive constant, and further the curvature $K_{t}$ of $\hat{S}_{t}$ satisfies: $1-\varepsilon(t) \leq K_{t} \leq 1+\kappa_{M}+\varepsilon(t)$, where $\varepsilon(t)$ goes to zero as $t \rightarrow \infty$, the first assertion (resp., the second assertion, the last assertion) turns out to be true because of the Rauch's comparison theorem (resp., the Klingenberg's theorem (cf. [4: Ch. 5]), Lemma 2 in [3]). This completes the proof of Corollary 2.4 .

Corollary 2.5. Let $M$ be as in Theorem B. Suppose that the dimension $m$ of $M$ is greater than or equal to 3 and for an end $\mathscr{E}_{\alpha}(M)$ of $M, \mathscr{V} o_{m-1}\left(M_{\alpha}(\infty)\right)$ is positive. Then $M_{\alpha}(\infty)$ is isometric to the space form of constant curvature 1 , if an integral $\int_{\delta_{\alpha}(M)}\left|\rho_{M}\right|^{p}(r+1)^{2 p-m}$ is finite for some $p \geq 1$, where $\rho_{M}$ (resp, $r$ ) denotes the scalar curvature of $M$ (resp. the distance to a fixed point).

Proof. In what follows, we assume for the sake of simplicity that $M$ has one end and we keep the same notations as in Lemma 2.3. Suppose that the integral $\int_{M}\left|\rho_{M}\right|^{p}(r+1)^{2 p-m}$ is finite for some $p \geq 1$. We observe that this is equivalent to the condition that the integral $\int_{M}\left\|R_{M}\right\|^{p}(r+1)^{2 p-m}$ is finite, since $\left\|R_{M}\right\| \leq c_{m}\left(\left|\rho_{M}\right|+k \circ r\right)$ for some constant $c_{m}$ depending only on $m$, where $R_{M}$ denotes the curvature tensor of $M$ and $k$ is as in (H.1). Then it follows that $\int_{A_{t}(a, b)}\left\|R_{t}\right\|$ converges to zero as $t \rightarrow \infty$, where $R_{t}$ is the curvature tensor of $(1 / t) M$. Therefore, applying the same argument of Lemma 1.2 to the family $\left\{A_{t}(a, b)\right\}$, we see that the cone $\mathscr{C}(M(\infty))$ is flat, nemely $M(\infty)$ has constant curvature 1 . This completes the proof of 
Corollary 2.5.

Remark. In Corollary 5.5, the condition: $\int_{\varepsilon_{\alpha}(M)}\left|\rho_{M}\right|^{p}(r+1)^{2 p-m}<+\infty$ can be replaced by a weaker one: $\lim \inf t^{2 p-m} \int_{A(a t, b t)}\left|\rho_{M}\right|^{p}=0$, where $A(a t, b t)=\{x \in M: b t \leq r(x) \leq a t\}(0<b<a)$.

Remark 2.6. Let $M$ be a manifold of asymptotically nonnegative curvarure. Then the condition (H.2), namely, the finiteness of $\kappa_{M}$, is obviously equivalent to the condition that the scalar curvature $\rho_{M}$ of $M$ satisfies: $\sup r^{2} \rho_{M}<+\infty$. Moreover given a nonnegative integer $k$, suppose that the Ricci tensor $S_{M}$ of $M$ satisfies: $\left\|\nabla^{i} S_{M}\right\| \leq c_{i} / r^{i+2}$ for each $i$ : $0 \leq i \leq k$ and some positive constants $c_{i}$. Then according to Theorem $\mathrm{A}^{\prime}$, Lemma 2.3 can be restated as follows: Fix two positive numbers $a, b$ with $a>b$. Then for large $t$, there exists a $C^{2+k}$-diffeomorphism $\Pi_{t}(0<\beta<1)$ from $A_{t}(a, b)$ into the cone $\mathscr{C}(M(\infty))$ over $M(\infty)$, i.e., $\mathscr{C}(M(\infty)):=[0, \infty)$ $\times_{t^{2}} M(\infty)$, which has the following properties: as $t$ goes to infinity, $\Pi_{t}\left(A_{t}(a, b)\right)$ converges to $[a, b] \times{ }_{t 2} M(\infty)$ and $\Pi_{t *} G_{t}$ converges to the metric $d t^{2}+t^{2} g_{\infty}$ in $C^{1+k, \beta^{\prime}}$ topology $\left(0<\beta^{\prime}<\beta<1\right)$, where $G_{t}$ (resp. $g_{\infty}$ ) is the Riemannian metric of $(1 / t) M$ (resp. $M(\infty))$.

\section{$\S 3$. The total curvature of a manifold of asymptotically nonnegative curvature}

A well known theorem of Cohn-Vossen [8] states that if the total

curvature integral $\int_{M} K_{M}$ on a complete oriented two dimensional Riemannian manifold $M$ is absolutely convergent, then $(1 / 2 \pi) \int_{M} K_{M} \leq \chi(M)$, where $K_{M}$ (resp., $\chi(M)$ ) denotes the Gaussian curvature (resp., the Euler characteristic) of $M$. Later, several authors, e.g., Huber [19, 20], Finn [11], Maeda [29], Shiohama [35, 36] etc., interpreted the difference: $\chi(M)-(1 / 2 \pi) \int_{M} K_{M}$. On the other hand, some authors, e.g., Greene-Wu [13], Poor [34], Walter [38] etc., made their attempts to generalize the Cohn-Vossen's inequality to 4- (or 6-) dimensional Riemannian manifolds of nonnegative sectional curvature (outside a compact set). The purpose of this section is to study some relations between the total curvature and the geometry at infinity of a manifold with asymptotically nonnegative curvature.

Throughout this section, $M$ is an oriented manifold of asymptotically 
nonnegative curvature and $m$ denotes the dimension of $M$.

3.1. To begin with, we shall prove the following

Proposition 4.1. Let $M$ be as above and suppose $m=2$, then the following assertions hold:

(i) The total curvature $\int_{M} K_{M}$ is absolutely convergent.

(ii) $\quad \chi(M)=(1 / 2 \pi) \int_{M} K_{M}+\operatorname{diam}(M(\infty)) / \pi$.

(iii) In the case: $K_{M} \geq 0$,

$$
2 \operatorname{diam}(M(\infty))=\inf _{p \in M} \operatorname{Length}\left(\mathscr{R}_{p}^{T}\right)=\inf _{D} \frac{[\operatorname{Length}(\partial D)]^{2}}{2 \operatorname{Area}(D)},
$$

where $\mathscr{R}_{p}^{T}:=\left\{v \in T_{p} M:|v|=1\right.$, $\exp _{p} t v \in \mathscr{R}_{p}$ (the set of all rays starting at $p$ ) $\}$ and $D$ ranges over all compact domains of $M$ with smooth boundary $\partial D$.

Proof. We shall prove the first assertion, referring to [24]. We write $K_{M}^{+}$(resp., $K_{M}^{-}$) for $\max \left\{0, K_{M}\right\}$ (resp., $\max \left\{0,-K_{M}\right\}$ ). Then observe first that the integral $\int_{M} K_{u}^{-}$is finite, because of Hypothesis (H.1) (cf. [24: 1.3]). We fix a point $p$ of $M$ and denote by $\widetilde{\mathscr{R}}_{p}^{T}$ the part of $\mathscr{R}_{p}^{T}$ which consists of a finite number of closed subarcs in the tangent unit circle parametrized by the arc-length $\theta(0 \leq \theta \leq 2 \pi)$. Then for sufficiently large $t$, we have

$$
\begin{aligned}
\chi(M) & =\chi\left(B_{t}(p)\right) \\
& =\frac{1}{2 \pi} \int_{B_{t}(p)} K_{M}+\frac{1}{2 \pi} \int_{\tilde{s}_{t}(p)} \kappa_{g, t}+\delta(t),
\end{aligned}
$$

where $\widetilde{S}_{t}(p):=S_{t}(p) \cap \exp _{p} t \widetilde{R}_{p}^{T}, \kappa_{g, t}$ is the geodesic curvature of $\widetilde{S}_{t}(p)$ and $\delta(t)$ goes to zero as $t \rightarrow \infty$ (cf. [24: Lemma 1.2 and Proposition 2.3]). Since $\liminf _{t \rightarrow \infty} \int_{\tilde{s}_{t}(p)} \kappa_{g, t} \geq 0$ (cf. [ibid.: Lemma 1.2 (iii)]), we have

$$
\int_{M}\left|K_{M}\right|<+\infty
$$

This proves the assertion (i). To show the second assertion, it is enough to prove that

$$
\int_{\tilde{S}_{t}(p)} \kappa_{g, t} \rightarrow 2 \operatorname{diam}(M(\infty))=\lim _{t \rightarrow \infty} \frac{\operatorname{Length}\left(S_{t}(p)\right)}{t}
$$

as $t \rightarrow \infty$. This is done as follows. For any $v(\theta)=(\sin \theta, \cos \theta) \in \widetilde{\mathscr{R}}_{p}^{T}$, set 
$J_{\theta}(t):=d \exp _{p \mid t v(\theta)} t(\partial / \partial \theta)$ (the Jacobi field along the ray $\exp _{p} t v(\theta)$ with $J_{\theta}(0)$ $=0$ and $J_{\theta}^{\prime}(0)=\partial / \partial \theta$ at $\left.v(\theta)\right)$. Then it turns out that $J_{\theta}(t)$ astisfies

$$
\left|J_{\theta}(t)\right|^{\prime \prime}+K_{M}\left(\exp _{p} t v(\theta)\right)\left|J_{\theta}\right|=0 .
$$

In partucular, we have

$$
\left|J_{\theta}(t)\right|^{\prime}=1-\int_{0}^{t} K_{M}\left(\exp _{p} s v(\theta)\right)\left|J_{\theta}(s)\right| d s .
$$

It follows from (3.1) that for almost all $v(\theta)$, the limit $\lambda(\theta)$ of $\left|J_{\theta}(t)\right|^{\prime}$ as $t \rightarrow \infty$ exists and it is given by

$$
\lambda(\theta)=1-\int_{0}^{\infty} K_{M}\left(\exp _{p} s v(\theta)\right)\left|J_{\theta}(s)\right| d s .
$$

Observe here that $\lambda(\theta)=\lim _{t \rightarrow \infty}\left|J_{\theta}(t)\right| / t$ for almost all $v(\theta)$. Therefore we get

$$
\begin{aligned}
\int_{\tilde{S}_{p}(t)} \kappa_{g, t} & =\int_{\tilde{\mathfrak{x}}_{p}^{T}}\left|J_{\theta}(t)\right|^{\prime} d \theta \rightarrow \int_{\tilde{\mathfrak{x}}_{p}^{T}} \lambda(\theta) d \theta \\
\frac{\operatorname{Length}\left(\tilde{S}_{p}(t)\right)}{t} & =\int_{\tilde{\mathfrak{x}}_{p}^{T}} \frac{\left|J_{\theta}(t)\right|}{t} d \theta \rightarrow \int_{\tilde{\mathfrak{x}}_{p}^{T}} \lambda(\theta) d \theta
\end{aligned}
$$

as $t \rightarrow \infty$. This shows (3.2), because $2 \operatorname{diam}(M(\infty))=\lim _{t \rightarrow \infty} \operatorname{Length}\left(\tilde{S}_{p}(t)\right) / t$ (cf. [24: Proposition 2.3]). Moreover it turns out from the above observation that

$$
2 \operatorname{diam}(M(\infty))=\operatorname{Length}\left(\widetilde{\mathscr{R}}_{p}^{T}\right)-\int_{\mathscr{P}_{p}} K_{M},
$$

where $\mathscr{D}_{p}:=\left\{\exp _{p} t v(\theta): t \geq 0, v(\theta) \in \widetilde{\mathscr{R}}_{p}^{T}\right\}$. Let us now suppose that $K_{M} \geq 0$ everywhere on $M$. Then we may assume that $M$ is diffeomorphic to $R^{2}$, because otherwise, $M$ is a flat cylinder $R \times S^{1}$. Taking account of the Toponogov's splitting theorem, we see that for any (small) number $\varepsilon>0$, there is a point $p_{\varepsilon}$ of $M$ with

$$
\int_{\mathscr{P}_{p_{\varepsilon}}} K_{M} \leq \varepsilon
$$

(cf. [29: p. 457]). Now it follows from (3.3) and (3.4) that for any $\varepsilon>0$,

$$
\begin{aligned}
\inf _{p \in M} \operatorname{Length}\left(\widetilde{\mathscr{R}}_{p}^{T}\right) & \geq 2 \operatorname{diam}(M(\infty)) \\
& \geq \operatorname{Length}\left(\widetilde{\mathscr{R}}_{p}^{T}\right)-\varepsilon \\
& \geq \inf _{p \in M} \operatorname{Length}\left(\widetilde{\mathscr{R}}_{p}^{T}\right)-\varepsilon .
\end{aligned}
$$


Thus we have shown that $2 \operatorname{diam}(M(\infty))=\inf _{p \in M}$ Length $\left(\widetilde{\mathscr{R}}_{p}^{T}\right)$. Finally we shall prove that $\operatorname{diam}(M(\infty))=I_{2}(M)^{2}$, where $I_{2}(M):=\inf \operatorname{Length}(\partial D) /$ Area $(D)^{1 / 2}$. This is a consequence of the second assertion (ii) as above and an isoperimetric inequality due to Fiala [10] and Huber [18]. Actually their isoperimetric inequality tells us that, for any simply connected domain $D$ of $M$,

$$
\frac{\operatorname{Length}(\partial D)^{2}}{4 \pi \operatorname{Area}(D)} \geq 1-\frac{1}{2 \pi} \int_{D} K_{M}^{+}
$$

Thus we have

$$
\begin{aligned}
\frac{\operatorname{diam}(M(\infty))}{\pi} & \geq \frac{I_{2}(M)^{2}}{4 \pi} \\
& \geq 1-\frac{1}{2 \pi} \int_{M} K_{M} \\
& =\frac{\operatorname{diam}(M(\infty))}{\pi} .
\end{aligned}
$$

Remarks. (i) The second assertion can be also derived from the first assertion and a result by Shiohama [35] (cf. also Finn [11] and Huber [20]).

(ii) Let $M$ be a manifold of asymptotically nonnegative curvature. Then as is mentioned in Introduction, for each end $\mathscr{E}_{\alpha}(M)$, we have the limit $\mathscr{V}_{o l_{m-1}}\left(M_{\alpha}(\infty)\right)$ of $\operatorname{Vol}_{m-1}\left(S_{t}(o) \cap \mathscr{E}_{\alpha}(M)\right) / t^{m-1}$ as $t \rightarrow \infty$, which is independent of the choice of the reference point $o$. Moreover it is not hard to see that

$$
\begin{aligned}
\mathscr{V} o l_{m-1}\left(M_{\alpha}(\infty)\right) & =\lim _{t \rightarrow \infty} \frac{m \operatorname{Vol}_{m}\left(B_{t}(o) \cap \mathscr{E}_{\alpha}(M)\right)}{t^{m}} \\
= & \lim _{t \rightarrow \infty} \frac{\left[\operatorname{Vol}_{m-1}\left(S_{t}(o) \cap \mathscr{E}_{\alpha}(M)\right)\right]^{m}}{\left[m \operatorname{Vol}_{m}\left(B_{t}(o) \cap \mathscr{E}_{\alpha}(M)\right]^{m-1}\right.} .
\end{aligned}
$$

Thus $\mathscr{V}_{o l_{m-1}}(M(\infty)):=\sum_{\alpha=1}^{\nu(M)} \mathscr{V} o l_{m-1}\left(M_{\alpha}(\infty)\right)$ is related to an isoperimetric constant $I_{m}(M)$ defined by

$$
I_{m}(M):=\inf \frac{\operatorname{Vol}_{m-1}(\partial \Omega)}{\left[\operatorname{Vol}_{m}(\Omega)\right]^{(m-1) / m}},
$$

where $\Omega$ ranges over all bounded domains of $M$ with smooth boundaries. Then, we have

$$
I_{m}(M) \leq m^{(m-1) / m}\left[\mathscr{V} o_{m-1}(M(\infty))\right]^{1 / m}
$$


Moreover if the Ricci curvature of $M$ is nonnegative everywhere, we see that

$$
I_{m}(M) \geq C(m)\left[\mathscr{V} o l_{m-1}(M(\infty))\right]^{1 / m},
$$

where $C(m)$ is a positive constant depending only on $m$. Actually, in this case, the measure of rays starting at $p$ with respect to the metric on the unit tangent sphere at $p$ is bounded from below by $\mathscr{V} o l_{m-1}(M(\infty))$, so that we can apply a theorem of Croke [7] to our situation.

3.2. As we have seen just above, the situation on $M$ is much simpler when the dimension $m$ is two. However it is more complicated in higher dimensional cases. In the next theorem, we consider the case as in Theorem B.

THeOREM 3.2. Let $M$ be a manifold of asymptotically nonnegative curvature satisfying the condition (H.2) as in Theorem B. Suppose that the dimension $m$ is greater than or equal to 3 . Then the following assertions hold:

(i) If the Gauss-Bonnet-Chern integral: $\int_{M} \Omega_{M}$ is absolutely convergent, then

$$
\left|\chi(M)-\int_{M} \Omega_{M}\right| \leq\left(1+\Lambda\left(m, \kappa_{M}\right)\right) \frac{\mathscr{V}_{o} l_{m-1}(M(\infty))}{\omega_{m-1}},
$$

where $\kappa_{M}$ is as in (H.2), $\Lambda\left(m, \kappa_{M}\right)$ is a nonnegative constant depending only on $m$ and $\kappa_{M}$, and $\omega_{m-1}$ denotes the volume of the unit $(m-1)$-sphere of Euclidean space. Moreover if $\kappa_{M}=0$, or $\int_{M}\left|\rho_{M}\right|^{p}(r+1)^{2 p-m}$ is finite for some $p \geq 1$, then

$$
\begin{aligned}
\chi(M) & =\int_{M} \Omega_{M}+\frac{\mathscr{V} o l_{m-1}(M(\infty))}{\omega_{m-1}} \\
& =\int_{M} \Omega_{M}+\sum_{\alpha} \frac{1}{\#\left(\pi_{1}\left(M_{\alpha}(\infty)\right)\right)},
\end{aligned}
$$

where the summation is taken over the ends $\mathscr{E}_{\alpha}(M)$ with $\mathscr{V} o_{m-1}\left(M_{\alpha}(\infty)\right)>0$.

(ii) The Gauss-Bonnet-Chern integral $\int_{M} \Omega_{M}$ is absolutely convergent, if one of the following conditions holds:

(ii-a) $m$ is odd $\left(\Omega_{M}: \equiv 0\right.$ by definition). 
(ii-b) $m=4$.

(ii-c) The total scalar curvature $\int_{M}\left|\rho_{M}\right|^{m / 2}$ is finite.

(ii-d) The sectional curvature of $M$ is nonnegative everywhere and $M$ is not diffeomorphic to $\boldsymbol{R}^{m}$ (in this case, $\mathscr{V} o_{m-1}(M(\infty))=0$ ).

(iii) In the case: $m=3,4$ or 5 , one has

$$
\chi(M)-\int_{M} \Omega_{M} \geq \frac{\mathscr{V}_{o} l_{m-1}(M(\infty))}{\omega_{m-1}} \geq 0 .
$$

Corollary 3.3. Let $M$ be as in Theorem 3.2 and suppose that $m$ is odd. Then one has the following assertions:

(i ) $\chi(M) \neq 0 \Rightarrow \mathscr{V} o l_{m-1}(M(\infty))>0$.

(ii) $\chi(M) \neq 0 \Longleftrightarrow \mathscr{V} o l_{m-1}(M(\infty))>0$, if $\kappa_{M}=0$, or $\int_{M}\left|\rho_{M}\right|^{p}(r+1)^{2 p-m}$ is finite for some $p \geq 1$.

(iii) $\chi(M) \neq 0 \Longleftrightarrow \mathscr{V} o_{m-1}(M(\infty))>0$ if $m=3$ or 5 .

(iv) $\chi(M) \neq 0 \Longleftrightarrow \mathscr{V}_{o l_{m-1}}(M(\infty))>0 \Longleftrightarrow M$ is diffeomorphic to $\boldsymbol{R}^{m}$, if $M$ has nonnegative sectional curvature.

Proof. The assertions (i)-(iii) are direct consequences of Theorem 3.2. The last one is derived from Theorem 3.2 and the structure theorem by Cheeger-Gromoll [6].

Corollary 3.4. Let $M$ be as in Theorem 3.2. Suppose that $m=4$ and

$$
\chi(M)-\frac{1}{2\left(\sqrt{\left.1+\kappa_{M}\right)^{3}}\right.}<\int_{M} \Omega_{M} .
$$

Then:

(i ) $\mathscr{V} o l_{3}(M(\infty))<\operatorname{Vol}_{3}\left(B_{r(M)}\left(1+\kappa_{M}\right)\right)$, where $r(M):=\pi / 2 \sqrt{1+} \overline{\kappa_{M}}$ and $B_{r}\left(1+\kappa_{M}\right)$ is the metric ball of radius $r$ in the 3-sphere of constant curvature $1+\kappa_{M}$.

(ii) $\operatorname{diam}\left(M_{\alpha}(\infty)\right)<\pi / 2$, if $\mathscr{\mathscr { V }} o l_{3}\left(M_{\alpha}(\infty)\right)>0$ for an end $\mathscr{E}_{\alpha}(M)$.

(iii) All of the Busemann functions on $M$ are exhaustion, if $\mathscr{V} l_{3}(M(\infty))$ $>0$ and the sectional curvature is nonnegative.

Proof. The first assertion is just a consequence of Theorem 3.2. The second one follows from the first one and Corollary 2.4(iii). The last one is derived from the second one and Corollary 4.4 in [24]. 
Proof of Theorem 3.2. To begin with, let us recall the definition of the Gauss-Bonnet-Chern integrand $\Omega_{M}$ of $M$ (cf. [7]). Let $\left\{e_{1}, \cdots, e_{m}\right\}$ be an orthonormal frame over a coordinate neighbourhood of $M$ and $\left\{\theta^{1}, \cdots\right.$, $\left.\theta^{m}\right\}$ the dual 1-forms. Then the structure equations on $M$ are given by

$$
d \theta_{i}=\omega_{i j} \wedge \theta^{j}, \quad d \omega_{i j}=\Omega_{i j}+\omega_{i k} \wedge \omega_{k j},
$$

where $\omega_{i j}$ and $\Omega_{i j}$ are, respectively, the connection 1-forms and the curvature 2 -forms of $M$. The Gauss-Bonnet-Chern form is the $m$-form on $M$ defined by

$$
\Omega_{M}:= \begin{cases}\frac{(-1)^{n}}{2^{m} \pi^{n} n !} \sum \varepsilon\left(i_{1}, \cdots, i_{m}\right) \Omega_{i_{1} i_{2}} \wedge \cdots \wedge \Omega_{i_{m-1} i_{m}} & (m=2 n) \\ 0 & (m=2 n+1),\end{cases}
$$

where the summation is taken over all permutations $\left\{i_{1}, \cdots, i_{m}\right\}$ of $\{1, \cdots, m\}$ and $\varepsilon\left(i_{1}, \cdots, i_{m}\right)$ is the signature. For an integer $k: 0 \leq k \leq \frac{1}{2}[m-1]$, define $(m-1)$-forms $\Pi_{k}$ on the tangent sphere bundle $S M$ of $M$ by

$$
\Pi_{k}(v)=C_{k, m} \sum \varepsilon\left(i_{1}, \cdots, i_{m-1}\right) \Omega_{i_{1} i_{2}} \wedge \cdots \wedge \Omega_{i_{2 k+1} i_{2 k}} \wedge \omega_{i_{2 k+1} v} \wedge \cdots \wedge \omega_{i_{m-1} v}
$$

for $v \in S M$, where the constants $C_{k, m}$ are given by

$$
C_{k, m}:= \begin{cases}\frac{(-1)^{k}}{\pi^{n} 2^{n+k} k !} \cdot \frac{1}{1 \cdot 3 \cdots(m-2 k-1)} & (m=2 n) \\
\frac{(-1)^{k+1}}{\pi^{n} 2^{m} n !}\left[\begin{array}{l}
n \\
k
\end{array}\right] & (m=2 n+1) .\end{cases}
$$

Let $D$ be a compact domain with smootk boundary $\partial D$. Then the GaussBonnet-Chern formula of [7] reads

$$
\chi(D)=\int_{D} \Omega_{D}-\sum_{k=0}^{[(m-1) / 2]} \int_{\partial D} \nu^{*} \Pi_{k},
$$

where $\nu$ denotes the unit outer normal to $\partial D$. Let $\left\{\lambda_{i}\right\}_{i=1, \ldots, m}$ be the eigenvalues of the second fundamental form $\alpha$ of $\partial D$ (i.e., $\alpha(X, Y):=\left\langle\nabla_{x} \nu, Y\right\rangle$ ). If we take an orthonormal frame $\left\{e_{1}, \cdots, e_{m}\right\}$ so that $\nu=e_{m}, \alpha\left(e_{i}, e_{j}\right)=$ $\lambda_{i} \delta_{i j}(i, j=1, \cdots, m-1)$ along $\partial D$, then the boundary integrands in (3.5) can be expressed as follows:

$$
\begin{aligned}
\nu^{*} \Pi_{k} & =(-1)^{m-1-2 k} C_{k, m} \sum \varepsilon\left(i_{1}, \cdots, i_{m-1}\right) \Omega_{i_{1} i_{2}} \wedge \cdots \wedge \Omega_{i_{2 k-1} i_{2 k}} \\
& \wedge \lambda_{i_{2 k+1}} \theta^{i_{2 k+1}} \wedge \cdots \wedge \lambda_{i_{m-1}} \theta^{i_{m-1}}
\end{aligned}
$$

Here it is convenient for us to introduce the following notations: for 
$t>0, M^{t}:=(1 / t) M, B^{t}:=$ the metric ball of $M^{t}$ around a fixed point of radius $1, S^{t}:=\partial B^{t}, \Omega^{t}:=$ the Gauss-Bonnet-Chern integrand of $M^{t}$, and $\Pi_{k}^{t}:=$ the $(m-1)$-form on the tangent sphere bundle of $M^{t}(0 \leq k \leq$ $[m-1] / 2)$. Since $S^{t}$ may not be smooth, we take the smooth approximation $\tilde{S}^{t}$ for $S^{t}$ (for large $t$ ) as in Theorem $B$. Then the second fundamental form $\tilde{\alpha}^{t}$ of $\tilde{S}^{t}$ satisfies

$$
\left|\tilde{\alpha}^{t}\right| \leq c\left(\kappa_{M}\right)+1
$$

for large $t$, where $c\left(\kappa_{M}\right)>0$ depends only on $\kappa_{M}$. Moreover in the case that $\mathscr{V} l_{m-1}\left(M_{\alpha}(\infty)\right)>0$ for some end $\mathscr{E}_{\alpha}(M)$, we may assume that

$$
(1-\varepsilon(t)) g_{t} \leq \tilde{\alpha}^{t} \leq(1+\varepsilon(t)) g_{t}
$$

on $\tilde{S}^{t} \cap \mathscr{E}_{a}(M)$, where $\varepsilon(t)$ goes to zero as $t \rightarrow \infty$ and $g_{t}$ is the Riemannian metric of $M^{t}$. Let us now apply the formula (3.5) to the domains $\tilde{B}^{t}$ which are bounded by $\tilde{S}^{t}$. Then since $M^{t}$ is isotopic to $\tilde{B}^{t}$, we have

$$
\begin{aligned}
\chi(M)=\chi\left(M^{t}\right) & =\chi\left(\tilde{B}^{t}\right) \\
& =\int_{\tilde{B}^{t}} \Omega^{t}-\sum_{\tilde{k}=0}^{[(m-1) / 2]} \int_{\tilde{S}^{t}} \nu_{t}^{*} \Pi_{k}^{t},
\end{aligned}
$$

where $\nu_{t}$ is the outer unit normal to $\tilde{S}^{t}$. The hypotheses (H.1), (H.2) and (3.6) imply that if the integral $\int_{M} \Omega_{M}$ is absolutely convergent, then

$$
\left|\chi(M)-\int_{M} \Omega_{M}\right| \leq\left(1+\Lambda\left(m, \kappa_{M}\right)\right) \cdot \frac{\mathscr{V} o l_{m-1}(M(\infty))}{\omega_{m-1}} .
$$

Suppose further that $\kappa_{M}=0$, or $\int_{M}\left|\rho_{M}\right|^{p}(r+1)^{2 p-m}$ is finite. Then $M_{\alpha}(\infty)$ has constant curvature 1 if $\mathscr{V} d_{m-1}\left(M_{\alpha}(\infty)\right)$ is positive, and we have

$$
\begin{aligned}
\chi(M) & =\int_{M} \Omega_{M}+\frac{\mathscr{V} o l_{m-1}(M(\infty))}{\omega_{m-1}}, \\
& =\int_{M} \Omega_{M}+\sum_{\alpha} \frac{1}{\#\left(\pi_{1}\left(M_{\alpha}(\infty)\right)\right)},
\end{aligned}
$$

because of (3.7) and the argument in the proof of Corollary 2.5. This proves the first assertion of Theorem 3.2.

Since $\int_{M}\left\|\Omega_{M}\right\| \leq c_{m}\left(\int_{M}\left|\rho_{M}\right|^{m / 2}+\int_{M} k \circ r^{m / 2}\right)$ for some constant $c_{m}$, the integral $\int_{M} \Omega_{M}$ is absolutely convergent, if the condition (ii-c) holds. In 
the case of (ii-d), $M$ has a soul of dimension $n>0$ and hence the volume element of $M$ grows like at most $r^{m-n}$ (cf. [22]). This shows that the integral $\int_{M} \Omega_{M}$ is absolutely convergent. Let us now examine more closely the cases: $m=3,4,5$. At first, we assume that $m=4$. In this case, we have

$$
\Omega_{M}=\frac{1}{4 \pi^{2}}\left\{R_{1212} R_{3434}+R_{1313} R_{2424}+R_{1414} R_{2323}+R_{1234}^{2}+R_{1324}^{2}+R_{4123}^{2}\right\} d v_{M}
$$

where $\Omega_{i j}=\frac{1}{2} R_{j i k \ell} \theta^{k} \wedge \theta^{\ell}$ and $d v_{M}:=\theta^{1} \wedge \theta^{2} \wedge \theta^{3} \wedge \theta^{4}$. If we put $\Omega_{\bar{M}}:=$ $\max \left\{0,-\Omega_{M} / d v_{M}\right\}$, then we have

$$
\Omega_{\bar{M}} \leq \frac{1}{\pi^{2}} K(r) k(r)
$$

where $k$ and $K(r)$ are as in (H.1) and (H.2), and $r$ is the distance to the base point $o$ in (H.1). Since the volume element $d v_{M}$ is estimated (within the cut locus of $M$ with respect to the reference point o) by $d v_{M} \leq J_{k}(r)^{3} d r$ $\wedge d \Theta$ in terms of the polar coordinates $(r, \Theta)$ around $o$, we have

$$
\int_{M} \Omega_{\bar{n}} d v<+\infty \text {. }
$$

Thus it is enough to show that

$$
\frac{\mathscr{V} o l_{3}(M(\infty))}{\omega_{3}} \leq \chi\left(\tilde{B}^{t}\right)-\int_{\tilde{B}^{t}} \Omega^{t}+\varepsilon(t),
$$

where $\varepsilon(t)$ goes to zero as $t \rightarrow \infty$. (3.8) is derived by the following observations:

$$
\begin{aligned}
& \lim _{t \rightarrow \infty}-\int_{\tilde{s}_{t}} \nu_{t}^{*} \Pi_{t}^{1}=\frac{\mathscr{V} o l_{3}(M(\infty))}{\omega_{3}} ; \\
& \liminf _{t \rightarrow \infty}-\int_{\tilde{s}^{t}} \nu_{t}^{*} \Pi_{1}^{t}=\liminf _{t \rightarrow \infty} \frac{1}{4 \pi^{2}} \int_{\tilde{s} t} \lambda_{1}^{t} R_{2323}^{t}+\lambda_{2}^{t} R_{1313}^{t}+\lambda_{3}^{t} R_{1212}^{t} \geq 0,
\end{aligned}
$$

where $\lambda_{i}^{t}(i=1,2,3)$ are the eigenvalues of $\tilde{\alpha}^{t}$.

We consider next the case: $m=5$. In this case, we have

$$
\begin{aligned}
& \lim _{t \rightarrow \infty}-\int_{\tilde{S} t} \nu_{t}^{*} \Pi_{0}^{t}=\frac{\mathscr{V} d_{4}(M(\infty))}{\omega_{4}} ; \\
& \liminf _{t \rightarrow \infty}-\int_{\tilde{s} t} \nu_{t}^{*} \Pi_{1}^{t}
\end{aligned}
$$




$$
\begin{aligned}
= & \liminf _{t \rightarrow \infty} \frac{1}{8 \pi^{2}} \int_{\tilde{s}^{t}}\left(\lambda_{3}^{t} \lambda_{4}^{t} R_{1212}^{t}+\lambda_{2}^{t} \lambda_{4}^{t} R_{1313}^{t}+\lambda_{2}^{t} \lambda_{3}^{t} R_{1414}^{t} \lambda_{1}^{t} \lambda_{4}^{t} R_{2323}^{t}+\lambda_{1}^{t} \lambda_{3}^{t} R_{2424}^{t}+\lambda_{1}^{t} \lambda_{2}^{t} R_{3434}^{t}\right) \\
\geq & 0 ; \\
& \liminf _{t \rightarrow \infty}-\int_{\tilde{s}^{t}} \nu_{t}^{*} \Pi_{2}^{t} \\
= & \liminf _{t \rightarrow \infty} \frac{1}{8 \pi^{2}} \int_{\tilde{S}^{t}}\left(R_{1212}^{t} R_{3434}^{t}+R_{1313}^{t} R_{2424}^{t}+R_{1414}^{t} R_{2323}^{t}+R_{1234}^{t}{ }^{2}+R_{1324}^{t}{ }^{2}+R_{1423}^{t}{ }^{2}\right) \\
\geq & 0 .
\end{aligned}
$$

These imply that $\chi(M) \geq \mathscr{V}_{o} l_{4}(M(\infty)) / \omega_{4}$.

Finally we assume that $m=3$. Then we have

$$
\begin{aligned}
& \lim _{t \rightarrow \infty}-\int_{\tilde{s} t} \nu_{t}^{*} \Pi_{0}^{t}=\frac{\mathscr{V} o l_{2}(M(\infty))}{\omega_{2}} ; \\
& \liminf _{t \rightarrow \infty}-\int_{\tilde{s} t} \nu_{t}^{*} \Pi_{1}^{t}=\liminf _{t \rightarrow \infty} \frac{1}{4 \pi} \int_{\tilde{s} t} R_{1212}^{t} \geq 0 .
\end{aligned}
$$

These shows that $\chi(M) \geq \mathscr{V}_{o} l_{2}(M(\infty)) / \omega_{2}$. This completes the proof of Theorem 3.2.

Remark. Let $M$ be a complete, minimal submanifold of dimension $m$ in Euclidean space $\boldsymbol{R}^{N}$. Suppose that the total scalar curvature: $\int_{M}\left|\alpha_{M}\right|^{m}$ is finite, where $\alpha_{M}$ denotes the second fundamental form of $M$. Then Anderson [1] shows that $\left|\alpha_{M}\right| \leq c / r^{m}$ for some positive constant $c$, where $r$ is the distance to a fixed point of $M$. In the case of $m \geq 3, M$ satisfies (H.1) and (H.2) with $\kappa_{M}=0$ and, since $M$ is a minimal submanifold of Euclidean space, $M_{\alpha}(\infty)$ is isometric to the unit $(m-1)$-sphere of $\boldsymbol{R}^{m}$ for each end $\mathscr{E}_{\alpha}(M)$. Thus it follows from Theorem 3.2 that $\chi(M)=\int_{M} \Omega_{M}+$ $\nu(M)$, where $\nu(M)$ stands for the number of the ends of $M$.

\section{§4. Gap Theorems for asymptotically flat manifolds}

A result of Greene-Wu [15] says that a complete, connected, noncompact Riemannian manifold $M$ of dimension $m$ must be isometric to Euclidean space $\boldsymbol{R}^{m}$, if $M$ is simply connected at infinity, the sectional curvature $K_{M}$ is everywhere nonnegative, and moreover (\#): $K_{M}$ vanishes outside a compact set. Here $M$ is called simply connected at infinity if for any compact set $C$, there is a compact set $C^{\prime}$ containing $C$ such that $M \backslash C^{\prime}$ is connected and simply connected. Moreover, Rəmark in p. 59 of 
Ballmann-Gromov-Schreoder [2] states (without proof) that the above fact still holds under a weaker condition: $\operatorname{dis}_{M}(x, o)^{2} K_{M}(x)$ goes to zero as $x \in M$ tends to infinity, instead of the condition (\#). We note that this stronger statement was actually proved by Greene-Wu [15] under the additional condition that $M$ possesses a pole $o$, namely, a point $o$ of $M$ where the exponential map $\exp _{o}: T_{o} M \rightarrow M$ induces a diffeomorphism. (They imposed there a further additional assumption on $M$ when $m=4$ or 8 , but it turns out to be a consequence from the other conditions. See [25, 26] for the reasoning and also more elementary proofs of the gap theorems in [15].) On the other hand, we have similar results for the cases of manifolds with nonpositive curvature (cf. [2: pp. 57-58] [15] [25, 26]).

In this section, we shall prove the following

THEOREM 4.1. Let $M$ be a manifold of asymptotically nonnegative curvature satisfying (H.2). Suppose that the dimension $m \geq 3$ and $\mathscr{V} o l_{m-1}(M(\infty))>0$, and suppose that $\kappa_{M}=0$, or $\int_{M}\left|\rho_{M}\right|^{p}(r+1)^{2 p-m}<+\infty$ for some $p \geq 1$. Then $M$ is isometric to Euclidean space $\boldsymbol{R}^{m}$ if one of the following conditions holds:

(i) The sectional curvature is nonnegative everywhere.

(ii) The Ricci curvature is nonnegative everywhere and $m$ is odd.

(iii). The Ricci curvature is nonnegative everywhere and $M$ is simply connected at infinity.

(iv) The sectional curvature is nonpositive everywhere.

Corollary 4.2. Let $M$ be a manifold of asymptotically nonnegative curvature satisfying (H.2). Suppose that the dimension $m$ is odd and the Euler characteristic $\chi(M)$ does not vanish, and suppose that $\kappa_{M}=0$, or $\int_{M}\left|\rho_{M}\right|^{p}(r+1)^{2 p-m}<+\infty$ for some $p \geq 1$. Then $M$ is isomorphic to Euclidean space $\boldsymbol{R}^{m}$ if the Ricci curvature is everywhere nonnegative, or if the sectional curvature is everywhere nonpositive.

Corollary 4.2 follows from Theorem 4.1 and Corollary 3.3.

Proof of Theorem 4.1. We first observe that if $m \geq 3$ and $\mathscr{V}_{o l_{m-1}}\left(M_{\alpha}(\infty)\right)$ $>0$ for some end $\mathscr{E}_{\alpha}(M)$, then $M_{\alpha}(\infty)$ is isometric to the quotient space of the unit sphere $S^{m-1}(1)$ of $R^{m}$ by a group $\Gamma_{\alpha}$ of isometries of $S^{m-1}(1)$ (cf. Corollary 2.5). Moreover it turns out from this observation, the assumption: $\mathscr{V} o l_{m-1}(M(\infty))>0$, and the splitting theorem by CheegerGromoll [5] that if the Ricci curvature of $M$ is nonnegative everywhere, 
then $M(\infty)$ is isometric to $S^{m-1}(1) / \Gamma$. On the other hand, we know that if the Ricci curvature of $M$ is nonnegative everywhere and $\mathscr{V}_{o l_{m-1}}(M(\infty))$ $=\omega_{m-1}\left(:=\operatorname{Vol}_{m-1}\left(S^{m-1}(1)\right)\right)$, then $M$ must be isometric to Euclidean space $\boldsymbol{R}^{m}$. Thus we have shown Theorem 4.1 under the condition (i) (cf. [6]), (ii) or (iii). It remains to prove the theorem under the condition (iv). Suppose the condition (iv) holds. We note then the following

FACT (cf. [25: Lemma 13]). Let $M$ be a complete, connected, and noncompact Riemannian manifold of nonpositive curvature. Suppose there is a compact subset $C$ of $M$ such that the fundamental group of a noncompact component of $M \backslash C$ is finite. Then $M$ is simply connected.

Thus it turns out that $M$ is simply connected, and hence $M$ is diffeomorphic to $\boldsymbol{R}^{m}$. This implies that $M(\infty)$ is isometric to $S^{m-1}(1)$, so that $M$ is isometric to $\boldsymbol{R}^{m}$, because of the Rauch comparison theorem.

Remark. Let $M$ be as in Theorem B and suppose the dimension is greater than or equal to 3 . Then very recently, Uesu [37] has proved that $M$ is isometric to Euclidean space if $M$ is simply connected and $M$ has no focal points.

\section{REFERENCES}

[1] M. T. Anderson, The compactification of a minimal submanifold in Euclidean space by the Gauss map, preprint.

[2] W. Ballmann, M. Gromov and V. Schroeder, Manifolds of Nonpositive Curvature, Progress in Math., 61, Birkhäuser, Boston-Basel-Stuttgart, 1985.

[ 3 ] D. L. Brittain, A diameter pinching theorem for positive Ricci curvature, preprint.

[ 4 ] J. Cheeger and D. G. Ebin, Comparison Theorems in Riemannian Geometry, NorthHolland, Amsterdam-Oxford-New York, 1975.

[5] J. Cheeger and D. Gromoll, The splitting theorem for manifolds of nonnegative Ricci curvature, J. Differential Geom., 6 (1971), 119-128.

[6] - On the structure of complete manifolds of nonnegative curvature, Ann. of Math., 96 (1974), 413-443.

[ 7 ] S.-S. Chern, On the curvature integra in a Riemannian manifold, Ann. of Math., 46 (1945), 674-684.

[8] S. Cohn-Vossen, Kurzeste Wege und Totalkrümung auf Flächen, Compositio Math., 2 (1935), 69-133.

[9] C. B. Croke, Some isoperimetric inequalities and eigenvalue estimates, Ann. Sci. Éc. Norm. Sup., Paris, 13 (1980), 419-435.

[10] F. Fiala, Le problème des isopérimètres sur les surfaces ouvertes à courbure positive, Comment. Math. Helv., 13 (1940/41), 293-346.

[11] M. Finn, On a class of conformal metrics, with application to differential geometry in the large, Comment. Math. Helv., 40 (1965), 1-30. 
[12] K. Fukaya, On a compactification of the set of Riemannian manifolds with bounded curvature and diameters, Curvature and Topology of Riemannian Manifolds, Lecture Notes in Math., 1201, Springer-Verlag, 1986.

[13] R. E. Greene and $\mathrm{H}$. Wu, $C^{\infty}$ convex functions and manifolds of positive curvature, Acta Math., 137 (1976), 209-245.

[14] _ - Function Theory on Manifolds Which Possess a Pole, Lecture Notes in Math., 699, Springer-Verlag, 1979.

[15] — Gap Theorems for noncompact Riemannian manifolds, Duke Math. J., 49 (1982), 731-756.

[16] - Lipschitz convergence of Riemannian manifolds, Pacific J. Math., 131 (1988), $119-141$.

[17] M. Gromov, Structures métrique pour les variétés riemanniennes, redige par J. Lafontaine et P. Pansu, Textes Math. No. 1, Edic/Fernand Nathan, Paris, 1981.

[18] A. Huber, On the isoperimetric inequality on surfaces of variable Gaussian curvature, Ann. of Math., 60 (1954), 237-247.

[19] - On subharmonic functions and differential geometry in the large, Comment. Math. Helv., 32 (1957), 13-72.

[20] — Métrique conformes complètes et singularités isolées de fonctions sousharmoniques, C. R. Acad. des Sci., Paris, 260 (1965), 6267-6268.

[21] J. Jost, Harmonic Mappings between Riemannian manifolds, Proc. Centre of Math. Analysis, Australia Nat. Univ., 4, 1983.

[22] A. Kasue, Applications of Laplacian and Hessian comparison theorems, Geometry of Geodesics and Related Topics, Advanced Studies in Pure Math., 3 (1984), 333386.

[23] — - On manifolds of asymptotically nonnegative curvature, preprint, M.S.R.I. Berkeley, July 1986.

[24] - A compactification of a manifold with asymptotically nonnegative curvature, Ann. Sci. Ecole Norm. Sup., Paris, 21 (1988), 593-622.

[25] A. Kasue and K. Sugahara, Gap theorems for certain submanifolds of Euclidean spaces and Hyperbolic space forms, Osaka J. Math., 24 (1987), 679-704.

[26] _ - _ II, Curvature and Topology of Riemannian Manifolds, Lecture Notes in Math., 1201, Springer-Verlag, 1986.

[27] A. Katsuda, Gromov's convergence theorem and its application, Nagoya Math. J., 100 (1985), 11-48.

[28] — A pinching problem for locally homogeneous spaces, Curvature and Topology of Riemannian Manifolds, Lecture Notes in Math., 1201, Springer-Verlag, 1986.

[29] M. Maeda, A geometric significance of total curvature on complete open surfaces, Geometry of Geodesics and Related Topics Advanced Studies in Pure Math., 3 (1984), 451-458.

[30] A. Nijenhuis-W. B. Woolf, Some integration problems in almost complex manifolds, Ann. of Math., 77 (1963), 424-483.

[31] L. G. Nikolaev, Smoothness of the metric of spaces with two-sided bounded Aleksandrov curvature, Siberian Math. J., 24 (1983), 247-263.

[32] S. Peters, Cheeger's finiteness theorem for diffeomorphism classes of Riemannian manifolds, J. reine angew. Math., 349 (1984), 77-82.

[33] — Convergence of Riemannian manifolds, Compositio Math., 62 (1987), 3-16.

[34] W. A. Poor, Jr., Some results on nonnegatively curved manifolds, J. Differential Geom., 9 (1974), 583-600.

[35] K. Shiohama, Total curvature and minimal areas of complete open surfaces, Proc. Amer. Math. Soc., 94 (1985), 310-316. 
[36] — An integral formula for the measure of rays on complete open surfaces, J. Differential Geom., 23 (1986), 197-205.

[37] K. Uesu, The Titz metric on focal points free manifolds and its application, preprint.

[38] R. Walter, A generalized Allendoeffer-Weil formula and an inequality of the CohnVossen type, J. Differential Geom., 10 (1975), 167-180.

Department of Mathematics

Osaka University

Toyonaka, Osaka 560, Japan 